\title{
GLI transcriptional repression is inert prior to Hedgehog pathway activation
}

Rachel K. Lex ${ }^{1}$, Weiqiang Zhou ${ }^{2}$, Zhicheng $\mathrm{Ji}^{2,3}$, Kristin N. Falkenstein ${ }^{1}$, Kaleigh E. Schuler ${ }^{1}$, Kathryn E. Windsor ${ }^{1}$, Joseph D. Kim ${ }^{1}$, Hongkai $\mathrm{Ji}^{2}$ and Steven A. Vokes ${ }^{1^{*}}$

${ }^{1}$ Department of Molecular Biosciences, University of Texas at Austin, 100 E 24th Street Stop A5000, Austin, TX 78712 USA

${ }^{2}$ Department of Biostatistics, Johns Hopkins Bloomberg School of Public Health, 615 North Wolfe Street, Room E3638, Baltimore, MD 21205, USA

${ }^{3}$ Current Address: Department of Biostatistics and Bioinformatics, Duke University School of Medicine, 2424 Erwin Road, Suite 1102 Hock Plaza Box 2721 Durham, NC 27710

"Corresponding author

Email: svokes@austin.utexas.edu

Phone: +1 512-232-8359 


\section{Summary}

In the absence of Hedgehog $(\mathrm{HH})$ signaling, GLI proteins are post-translationally modified within cilia into transcriptional repressors that subsequently prevent sub-threshold activation of $\mathrm{HH}$ target genes. GLI repression is presumably important for preventing precocious expression of target genes before the onset of $\mathrm{HH}$ pathway activation, a presumption that underlies the prepatterning model of anterior-posterior limb polarity. Here, we report that GLI3 repressor is abundant and binds to target genes in early limb development. However, contrary to expectations, GLI3 repression neither regulates the activity of GLI enhancers nor expression of $\mathrm{HH}$ target genes as it does after $\mathrm{HH}$ signaling has been established. Within the cilia, the transition to active GLI repression is accompanied by increases in axonemal GLI3 localization, possibly signifying altered GLI3 processing. Together, our results demonstrate that GLI3 repression does not prevent precocious activation of $\mathrm{HH}$ target genes, or have a pre-patterning role in regulating anterior-posterior limb polarity.

\section{Keywords}

Hedgehog signaling, GLI, GLI3, transcriptional repression, chromatin, limb bud, limb development, pre-patterning, cilia

\section{Introduction}

The Hedgehog $(\mathrm{HH})$ signaling pathway is one of the major developmental regulators of tissuespecific development and differentiation. GLI proteins mediate transcriptional responses to the pathway in a strikingly cell-specific fashion. In the absence of $\mathrm{HH}$ pathway activation, GLI transcriptional repressors (GLI-R) prevent the activation of $\mathrm{HH}$ target genes, while upon exposure to $\mathrm{HH}, \mathrm{GLI}$ proteins undergo alternative post-translational processing into transcriptional activators (GLI-A) (Aza-Blanc et al., 1997; Méthot and Basler, 1999; Panman et al., 2006; Wang et al., 2000; Niewiadomski et al., 2014). The processing of GLI-A and GLI-R are 
both directly dependent on processes localized within the primary cilium and defects in ciliary components lead to characteristic misregulation of $\mathrm{HH}$ target genes in a large group of birth defects termed ciliopathies (reviewed in Ho and Stearns, 2021). While GLI-A directly activates a subset of $\mathrm{HH}$ targets, most genes are 'de-repressed' by the loss of GLI-R alone; they do not require GLI-A-mediated activation for their expression. The importance of de-repression is exemplified in the developing limb where the phenotype of Sonic Hedgehog (Shh) null limb buds is dramatically improved in Shh;Gli3 mutants (Lewandowski et al., 2015; Litingtung et al., 2002; te Welscher et al., 2002). In this context, the loss of GLI3-mediated repression, even in the absence of pathway activation, is sufficient to restore expression of most GLI target genes and many aspects of limb growth and patterning.

GLI3 represses transcription at least in part by epigenetically regulating a subset of its own enhancers. Its properties include reduced enrichment levels of the active enhancer mark $\mathrm{H} 3 \mathrm{~K} 27 \mathrm{ac}$ and reduced chromatin accessibility at a subset of $\mathrm{HH}$-responsive GLI3 binding regions (GBRs), termed $\mathrm{HH}$-responsive GBRs, that likely mediate the majority of $\mathrm{HH}$-specific transcription (Lex et al., 2020). As a regulator of tissue patterning, GLI-R spatially and temporally restricts expression of $\mathrm{HH}$ targets, preventing sub-threshold activation of the pathway in HH-responsive tissues (Aza-Blanc et al., 1997; Balaskas et al., 2012; Parker et al., 2011). Consequently, GLI transcriptional repression has primarily been studied in tissues with ongoing $\mathrm{HH}$ signaling. Although never experimentally addressed, it is widely assumed that GLI-R also regulates the expression of its target genes prior to the initiation of $\mathrm{HH}$ signaling. Before pathway activation, GLI3 has been proposed to have a pre-patterning role in the very early limb by repressing Hand2, thus limiting its expression to the posterior limb bud where it is required to activate Shh expression and establishing anterior-posterior polarity of the limb (Galli et al., 2010; Litingtung et al., 2002; Osterwalder et al., 2014; Vokes et al., 2008; te Welscher et al., 2002; Zhulyn et al., 2014; reviewed in Zuniga and Zeller, 2020). Curiously, Hand2 and Gli3 are co- 
expressed in early limb buds, prior to their segregation into distinct posterior and anterior domains (Osterwalder et al., 2014), raising the question of whether GLI3-R is capable of repressing Hand2 at this time.

We initially hypothesized that GLI3-mediated repression would be important prior to $\mathrm{HH}$ expression for preventing premature activation of target genes by reducing H3K27ac enrichment at enhancers. Despite GLI3 binding to a majority of the same sites it occupies in the post-HH limb bud, it does not regulate activation of its enhancers or their chromatin accessibility until after the initiation of $\mathrm{HH}$ signaling. In addition, GLI target genes are not upregulated in limb buds lacking GLI3 as they are after the initiation of $\mathrm{HH}$ signaling, even though a subset of these genes appear competent to be repressed at this stage. Counter to our initial hypothesis, we conclude that the GLI3-R isoform is transcriptionally inert in early limb buds, a finding that is incompatible with the pre-patterning model for limb polarity. Interestingly, most genes subject to later GLI3 mediated repression are expressed in pre-HH limb buds, suggesting that rather than preventing their activation, GLI3 repression later regulates their spatial expression. Overall, this work demonstrates that GLI3-mediated repression of target genes is not a default state, but rather, GLI repression is established during limb development at a time point after $\mathrm{HH}$ induction.

\section{Results}

\section{GLI3 repressor is abundant and binds to most regions prior to $\mathrm{HH}$ induction}

To understand if GLI repression is established prior to $\mathrm{HH}$ signaling we first defined when the pathway is activated during limb development. The earliest detection of the canonical $\mathrm{HH}$ target gene Gli1 was at 24 somites (24S), where $58 \%$ of embryos at this stage had detectable Gli1 expression, while Shh was not detected until 25S (86\%) (Figure 1A,B; Figure S1A,B). As 24S 
was the earliest detection of Gli1 expression, we defined that stage as the onset of $\mathrm{HH}$ signaling and the "pre-HH" window as 21-23S, corresponding to embryonic day 9.25 (E9.25), a stage slightly earlier than previous reports (Figure 1A) (Charite et al., 2000; Lewis et al., 2001; Zhu et al., 2008).

Confirming previous findings, GLI3-R was expressed at comparable levels at both pre- and post-HH stages (Figure 1C) (Osterwalder et al., 2014). We then compared endogenous GLI3 ${ }^{\text {FLAG }}$ binding using CUT\&RUN (Skene and Henikoff, 2017) in pre-HH (E9.25, 21-23S) and E10.5 (32-35S) limb buds, a post-HH time frame when $\mathrm{HH}$ signaling is firmly established but before there are morphological changes in Shh ${ }^{-/}$limb buds (Probst et al., 2011). In pre-HH limb buds, GLI3 bound to most regions that were bound in post-HH limb buds (82\%; Figure 1D). Previously, we identified a group of HH-responsive GLI3 binding regions that have reduced $\mathrm{H} 3 \mathrm{~K} 27 \mathrm{ac}$ in the absence of $\mathrm{HH}$ signaling (constitutive GLI repression) (Lex et al., 2020)(Figure S1C-E). Since this subset of GBRs seems to regulate most $\mathrm{HH}$-responsive gene expression, we reasoned that these regions might be especially important to repress before $\mathrm{HH}$ signaling to prevent premature activation of enhancers. While the majority of $\mathrm{HH}$-responsive GBRs (70\%) were bound by GLI3 prior to $\mathrm{HH}$ induction, nearly a third were not bound, suggesting that GLI3 does not initially regulate a subset of $\mathrm{HH}$-responsive GBRs (Figure 1E).

\section{GLI3 preferentially binds to poised, accessible enhancers}

We sought to determine why GLI3 bound to only a portion of regions in the pre-HH limb and hypothesized that GLI3 may preferentially bind to poised enhancers. We defined poised enhancers as ATAC-seq accessible and enriched for either H3K4me1 or H3K4me2, where $\mathrm{H} 3 \mathrm{~K} 4 \mathrm{me} 1$ is enriched at promoter-proximal and distal regions, while $\mathrm{H} 3 \mathrm{~K} 4 \mathrm{me} 2$ is more commonly found promoter proximally (Ernst et al., 2011; Pekowska et al., 2011; Wang et al., 
2014). Most HH-responsive GBRs that were accessible and enriched for H3K4me2 by E10.5 were already accessible (89\%) and enriched for H3K4me2 (98\%) in the early limb (Figure S1F,G). In contrast, of the E10.5 HH-responsive GBRs with H3K4me1, only $65 \%$ of them were enriched for H3K4me1 by E9.25 (Figure S1G). We then asked if GLI3 preferentially bound to accessible, poised regions. Consistent with this scenario, most regions bound by GLI3 in pre$\mathrm{HH}$ limbs were accessible (93\%) and enriched for poised enhancer modifications (75\%) (Figure 1F,G; Figure S1G). These included a defined limb-specific distal enhancer for Ptch1 (LopezRios et al., 2014) (Figure 1H). While nearly half of the regions not yet bound by GLI3 in the early limb overlapped with called ATAC-seq peaks (Figure S1H), they were less accessible than GLI bound enhancers (Figure 1G). Unbound HH-responsive GBRs also generally lacked enrichment of poised enhancer marks, H3K4me1 and H3K4me2 (Figure 1G,I; Figure S1H). As many distal regions lacked $\mathrm{H} 3 \mathrm{~K} 4 \mathrm{me} 1$ enrichment prior to $\mathrm{HH}$ induction, we observed a slight preference for GLI3 to be bound to promoter proximal regions (Figure S1I). This is exemplified by the distal limb enhancer, GRE1 that helps regulate the $\mathrm{HH}$ target Gremlin, which is among the inaccessible regions that lack H3K4me1 enrichment and are not bound by GLI3 at E9.25 (Figure 1I). This finding is consistent with previous reports demonstrating that GRE1 does not have enhancer activity until E10 (31-32S) (Li et al., 2014). We conclude that the majority of GLI enhancers have accessible chromatin in pre-HH limb buds. In addition, we indicate that GLI3 preferentially binds to poised enhancers, providing tissue-specific control of repression.

\section{HH-responsive GBRs have low levels of H3K27ac and are enriched with HDACs, before activation of the $\mathrm{HH}$ pathway}

If GLI3 represses enhancers in the early limb as it does at E10.5, then HH-responsive GBRs, which have reduced $\mathrm{H} 3 \mathrm{~K} 27 \mathrm{ac}$ in $\mathrm{E} 10.5 \mathrm{Shh}^{-/}$limb buds, should have reduced acetylation at 
E9.25 before $\mathrm{HH}$ signaling initiates. In agreement with this scenario, there was a significant reduction in $\mathrm{H} 3 \mathrm{~K} 27$ ac enrichment at $\mathrm{HH}$-responsive GBRs in pre-HH compared to post-HH limb buds (Figure 2A-C). In addition, only 39\% (121/309) of HH-responsive GBRs have called H3K27ac peaks in the pre-HH limb, compared to 86\% (6385/7382) of all GBRs that are acetylated in the early limb bud. The overall reduction in H3K27ac enrichment at HH-responsive GBRs in the early limb bud supports the possibility that GLI3 could be actively repressing enhancers at this time to prevent premature activation.

Previously, we found that GLI-mediated repression is facilitated by HDACs that regulate H3K27ac enrichment at GBRs (Lex et al. 2020). To determine whether GLI3 binding is associated with HDACs in pre-HH limb buds, we performed CUT\&RUN in pre- and post-HH limbs with antibodies against HDAC1 and HDAC2. Pre-HH limb buds had similar levels of HDAC1 and HDAC2, with many GBRs enriched for both HDACs (Figure 2D,E). In post-HH limb buds, most of these regions continue to be bound by HDAC1 while HDAC2 binding is greatly reduced (Figure 2F). Overall, the presence of HDACs at most GBRs prior to $\mathrm{HH}$ is consistent with a model in which GLI3, together with an HDAC-containing repression complex, could be repressing enhancers to prevent premature activation of target genes (Figure 2G).

\section{GLI3 enhancers are unable to be prematurely activated with loss of Gli3}

We next tested the hypothesis that the reduction in acetylation at GBRs prior to $\mathrm{HH}$ signaling was due to GLI3-mediated repression which reduces the levels of H3K27ac, preventing premature activation of enhancers (Figure 2G). We examined H3K27ac enrichment in WT and

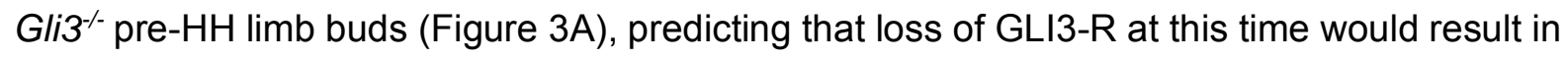
increased acetylation at GBRs, as it does in E10.5 post-HH limbs (Lex et al., 2020) (Figure 3B). Contrary to our hypothesis, there was no significant increase in H3K27ac enrichment in Gli3 ${ }^{-1}$ 
limb buds prior to $\mathrm{HH}$ induction (Figure 3B-F). Since GLI3-mediated repression of Hand2 in the early limb is a key component of the pre-patterning model of anterior-posterior polarity (Litingtung et al., 2002; Osterwalder et al., 2014; Panman and Zeller, 2003; Welscher et al., 2002; Zhulyn et al., 2014; Bénazet and Zeller, 2009; Galli et al., 2010), we examined two GLI3 binding regions located $10 \mathrm{~kb}$ and $85 \mathrm{~kb}$ downstream of Hand2 that have been suggested to mediate GLI3 repression (Vokes et al., 2008), but did not observe any increases in acetylation (Figure 3E, Figure S2A). We also considered the possibility that many enhancers, which have low levels of $\mathrm{H} 3 \mathrm{~K} 27 \mathrm{ac}$, may not be competent for activation at this stage and would therefore not have increased enrichment of H3K27ac upon loss of Gli3. To address this, we examined three outlier $\mathrm{HH}$-responsive GBRs that contained significantly higher levels of H3K27ac in pre$\mathrm{HH}$ limb buds (Figure 2B) to see if the acetylation levels might further increase in the absence of GLI3 repression. Despite GLI3 binding at these regions, there was no increase in H3K27ac enrichment with loss of Gli3 before $\mathrm{HH}$ signaling (Figure S2B-D). Overall, this surprising result indicates that despite the presence of GLI3 and HDACs, GLI repression does not regulate H3K27ac enrichment at enhancers in pre-HH limb buds (Figure 3F).

\section{Most GLI3 target genes are unable to be prematurely activated with loss of Gli3}

To determine whether the lack of enhancer activation in E9.25, pre-HH Gli3 ${ }^{-/}$limbs corresponded with a lack of de-repression of GLI target genes, we performed bulk RNA-seq on pre-HH WT and Gli3 ${ }^{-/}$limbs, as well as the anterior halves of post-HH (32-35S) WT and Gli3 ${ }^{-/}$ limbs (Figure 4A-D). In post-HH Gli3 ${ }^{-/}$limbs, we identified a total of 159 significantly upregulated genes (FDR<0.05) that included well known signature $\mathrm{HH}$ target genes Ptch1, Hand2, Hoxd13 and Grem1 (Figure 4C,D) (Lewandowski et al., 2015). However, in agreement with the lack of GLI3-regulated enhancer activity, very few genes (9) were significantly upregulated with loss of 
Gli3 in the early limb (Figure 4B). These genes include Foxf1 and Osr1, which are regulated by $\mathrm{HH}$ signaling in multiple mesodermal tissues but, with the exception of Gpx6, are not upregulated in E10.5 Gli3 ${ }^{-/}$limbs (Guzzetta et al., 2020; Hoffmann et al., 2014; Mahlapuu et al., 2001; Nasr et al., 2020). This suggests that rather than being a product of ongoing GLI3 repression, these upregulated genes may either represent contaminating flank tissue from the dissection. Alternatively, these could be residual mRNAs upregulated prior to limb bud initiation, as limb outgrowth requires cells from the lateral plate mesoderm to undergo an epithelial to mesenchymal transition which would have occurred within the last $\sim 8$ hours (Gros and Tabin, 2014). Consistent with the former possibility, Foxf1 is present in the lateral plate mesoderm but is not detected in either WT or Gli3-/ pre-HH limb buds (Figure 4D). Similarly, Ptch1, a signature $\mathrm{HH}$ target gene was detected in the neural tube, but was also not detected in the early limb buds in WT or Gli3/-- embryos (Figure 4D). In support of the latter possibility, pre-HH upregulated mRNAs are reported to have an average half-life of 11.2 hours in differentiating ES cells (Figure S3A) (Sharova et al., 2009), and also have lower levels of retained introns than post-HH upregulated $\mathrm{Gli3}^{-/}$genes, suggesting that some of the these transcripts may represent older mRNAs, potentially regulated by GLI3 in the lateral plate mesoderm, that are not currently being transcribed in the limb (Figure S3B,C). We conclude that GLI3 is unlikely to be engaged in ongoing transcriptional repression in the early limb bud.

As Hand2 is known to be repressed by GLI3 in early limb buds, we examined Hand2 expression in pre-HH and early post-HH WT and Gli3-- embryos to look for subtle changes that might not be detected by RNA-seq. Hand2 is initially expressed throughout anterior WT limb buds (Figure 4D,E) similar to Gli3-/ limb buds (20-23S) ( $n=7)$. At early post-HH stages (24-26S), Hand2 expression is variable in WT and Gli3 ${ }^{-/}$limb buds, with most WT limb buds expressing anterior Hand2 and lower levels of anterior Hand2 expression present even in some Gli3 $^{-}$limb buds (Figure 4E, white arrow). Posterior restriction of Hand2 is not evident until $26 \mathrm{~S}$ and is still 
variable at that time (Figure 4E, Figure S3D). Our findings are consistent with previous reports that noted early co-expression of Gli3 and Hand2 at these stages (Osterwalder et al., 2014). We conclude that GLI3 repression of Hand2 is absent prior to the activation of $\mathrm{HH}$ signaling and suggests that GLI3 repression is first activated between 24-26S, coinciding with the initiation of $\mathrm{HH}$ signaling (Figure S1A,B).

\section{GLI3 repressor is inert in early limb buds}

Our results thus far indicated that GLI repression is not active in the early limb bud. This could either be caused by inert GLI repression complexes or by target genes that were incapable of being activated. To understand whether PRC2 might broadly regulate $\mathrm{HH}$ targets, we computationally predicted $74 \mathrm{GLI}$ target genes through identifying genes downregulated in $S h h^{-/}$ limbs that were near HH-responsive GBRs (Supplemental Table 1)(Lewandowski et al., 2015). We performed CUT\&Tag (Kaya-Okur et al., 2019) for H3K27me3, a modification indicative of PRC2 repression in pre-HH and post-HH limb buds (Figure 4F) (Margueron and Reinberg, 2011). There was little H3K27me3 enrichment at target gene promoters after $\mathrm{HH}$ induction, with the notable exceptions of Ptch1 and Gli1 in anterior limbs, consistent with previous reports (Lex et al., 2020) (Figure 4H,I; Figure S4A-D; Supplemental Table 1). However, in pre-HH limb buds, $81 \%$ of putative $\mathrm{HH}$ target gene promoters were enriched for H3K27me3 (Figure S4A). This H3K27me3 enrichment appears to resolve over limb development, as many genes completely lose H3K27me3 enrichment or have drastic reductions by E10.5, with only $21 \%$ remaining trimethylated in anterior limbs (Figure 4H; Figure S4A-D). Most HH-responsive GBRs lacked H3K27me3 enrichment, while the few GBRs enriched for H3K27me3, were generally located proximal to promoters (Figure 4I; Figure S4B). There were high levels of H3K27me3 at the Hand2 locus in the early limb (Figure S4F), which as noted above is widely expressed 
throughout the limb at this time. Hand2 also was enriched for H3K27ac, which is mutually exclusive with H3K27me3 (Zhang et al., 2015), suggesting that PRC2 is associated with Hand2 in a subset of cells that presumably do not express Hand2 in pre-HH limb buds (Figure S4F). Similar to Hand2, nearly half of predicted $\mathrm{HH}$ target genes were enriched for both H3K27ac and H3K27me3, which was also true of the few E10.5 genes enriched for H3K27me3 (Figure S4D; Supplemental Table 1). An important distinction between the two developmental stages is that at E9.25, most genes have high levels of H3K27me3 and low levels of H3K27ac, while at E10.5 when trimethylation is resolving, most genes have increased levels of H3K27ac with low levels of $\mathrm{H} 3 \mathrm{~K} 27 \mathrm{me} 3$, consistent with increases in their expression levels at E10.5 (Figure S4D-G). For other genes enriched for both marks, like Ptch1, the distribution of these modifications is offset, supporting the potential for these mutually exclusive marks to be present at the same locus (Figure 4I, black brackets). The dual enrichment of H3K27ac and H3K27me3 may enable fast induction of these genes upon presence of relevant stimuli, while preventing inappropriate expression of them from non-relevant stimuli, as others have proposed (Kitazawa et al., 2021). A smaller population of genes (18\%), are highly enriched for H3K27me3 and completely lack acetylation. Based on these findings, at least some $\mathrm{HH}$ target genes with $\mathrm{H} 3 \mathrm{~K} 27 \mathrm{me} 3$ enrichment at this stage may not be competent for de-repression upon loss of Gli3 (Figure 4I) and GLI3 repression itself is unlikely to facilitate $\mathrm{H} 3 \mathrm{~K} 27 \mathrm{me} 3$ enrichment (see discussion). Importantly, a third of genes are bound by GLI3 with no H3K27me3 enrichment and are likely to be competent for de-repression upon the loss of Gli3. However, as we did not observe increased acetylation in E9.25 $\mathrm{Gli3}^{-/-}$limbs at these regions as we do in E10.5 $\mathrm{Gli3}^{-/-}$limb buds, we conclude that GLI3 repressor is inert in the early limb bud (Figure 4J). 


\section{GLI3-dependent chromatin compaction does not initiate until after the start of $\mathrm{HH}$} signaling

To define the onset of GLI repression, we looked for evidence of GLI3 repression at 28-30S (E10), about 8-12 hours after $\mathrm{HH}$ activation. We used chromatin accessibility as a read out for GLI repression, as E10.5 (35S) Shh- limb buds (constitutive GLI repression) have reduced accessibility at HH-responsive GBRs compared to WT limb buds (Lex et al., 2020). To verify that this chromatin compaction was mediated through GLI repression and not lack of GLI activator, we performed ATAC-seq in Shh ${ }^{-/} ;$Gli3 $^{-/-}$E10.5 limb buds. Loss of Gli3 resulted in accessible chromatin at HH-responsive GBRs similar to WT controls, confirming that chromatin compaction is GLI3 repressor-dependent (Figure 5A).

As Hand2 expression becomes posteriorly restricted in the limb around 26S, we anticipated that GLI3 repression should be established by $28-30$ S (E10), and predicted that chromatin in Shh"limbs at this stage would be more compacted compared to WT. Alternatively, if GLI-repression was yet to become established, or was still in the process of being established, the accessibility of $\mathrm{HH}$-responsive GBRs would be similar to that of E10 WT limbs. Consistent with the latter scenario, the chromatin at $\mathrm{HH}$-responsive GBRs overall was not compacted. In fact, it was significantly more accessible in E10 Shh ${ }^{-/}$limb buds compared with E10 WT limbs (Figure 5AD). This surprising result indicated that one of the signatures of GLI repression had largely not been established in this population at this stage in development. We next examined individual GBRs to determine if there were any regions with less accessible chromatin in E10 Shh ${ }^{-/-} \operatorname{limb}$ buds, indicative of initial targets of GLI3 repression. While most regions were not significantly reduced, several regions have a trend toward having reduced accessibility at E10 in Shh ${ }^{-/}$ compared to WT, such as HH-responsive GBRs near HH targets Ptch1 and Hhip. However, this reduction did not become significant until E10.5 (Figure 5E-G, blue datapoints). Only a few HHresponsive GBRs had significantly reduced accessibility in E10 in Shh/ limb buds including 
GBRs around Cdh11 and MIlt3, which were also significantly reduced at E10.5 (Figure 5E,F,H; Supplemental File 4). Collectively these results support that GLI repression is not fully established even up to 12 hours after $\mathrm{HH}$ signaling would have normally become active (Figure 5J).

\section{Changes in GLI3 ciliary distribution correspond with the onset of GLI3-dependent chromatin compaction}

As GLI3 repression does not initiate until after the induction of $\mathrm{HH}$ signaling, we asked if there was a change in the ciliary trafficking of GLI3 proteins that could signify changes in the processing of GLI3. In pre-HH limb buds, $\sim 80 \%$ of cilia contained endogenous GLI3 ${ }^{\text {FLAG }}$ (Figure $\mathrm{S} 5 \mathrm{~A}, \mathrm{~B}, \mathrm{E})$. There were similarly high trends in both GLI3, as well as GLI2 ciliary co-localization in pre-HH WT and $S h h^{-/}$limb buds, where expression was primarily localized to the ciliary tip (Fig. S5A-E). This indicates that this enrichment is not due to undetectable levels of $\mathrm{SHH}$ that might still promote GLI activation and, as GLI-R is not localized to the cilia, it suggests that pre-HH ciliary localization is enriched with full-length, unprocessed GLI protein (Figure S5A-E) (Haycraft et al., 2005; Wen et al., 2010). While GLI ciliary localization remained at comparable levels shortly after the onset of SHH expression (E9.75, 26-28S) as well as at E10.5 (32-35S; Fig. S5F), E9.75 limb buds had a significant redistribution of GLI3 within the cilia, with $74 \%$ of cilia having GLI3 signal along the ciliary axoneme and a corresponding reduction in the percentage of cilia with tip-restricted GLI3 expression (Fig. 5K, L; Fig. S5G). Overall, this data is consistent with the possibility that there may be a 'steady state' of low to moderate levels of GLI3 ciliary trafficking and processing, with a spike in the rate of trafficking coinciding with the requirement for GLI3 repression to become established during limb development. 


\section{GLI3 is unlikely to co-regulate HAND2 targets in early limb buds despite co-expression}

GLI3-repression of Hand2 is initiated concomitantly with activation of $\mathrm{HH}$ signaling and therefore GLI3 does not have a bona-fide pre-patterning role. In pre- and post-HH limb buds, GLI3 and HAND2 bind to nearly adjacent regions in IRX3/5 as well as many other targets (Osterwalder, 2014; Vokes et al, 2008), suggesting that they might co-regulate a common set of binding regions. (Figure S6A) (Osterwalder et al., 2014). Interestingly, HAND2 and GLI3 have been shown to bind to each other and to synergistically activate targets through common CRMs in developing facial elements (Elliott et al., 2020). While HAND2 and GLI3 are mutually exclusive in later limb buds (teWelscher et al, 2002; Osterwalder et al, 2014), their co-occurrence in pre$\mathrm{HH}$ limb buds suggests the possibility that GLI3-R could facilitate HAND2 regulation of target genes in its capacity as a DNA binding protein rather than as a repressor. In support of this possibility, HAND2-bound enhancers for Tbx2 and Tbx3 contain GBRs that have called GLI3 peaks in pre-HH but not post-HH limb buds (Figure S6B, light blue shading). To determine whether GLI3 and HAND2 might initially co-activate targets in the early limb in a similar fashion to craniofacial tissue, we identified a population of 310 competent GBRs specifically bound by GLI3 at E9.25, but not at E10.5, that also overlap previously identified HAND2 limb binding sites (Figure S6B,C)(Osterwalder et al., 2014). We analyzed previously reported HAND2 and GLI3 motifs (Elliott et al., 2020; Lex et al., 2020) in ATAC-seq footprints in the early limb compared to post-HH Shh ${ }^{-/}$limbs, which have active GLI repression and would not be expected to have HAND2 and GLI3 co-regulation. If GLI3 and HAND2 were to coordinate enhancer activation, we predicted HAND2 motifs would be enriched in pre-HH limbs compared to post-HH Shh ${ }^{-/}$limbs. Inconsistent with this scenario, there was no difference in HAND2 motif enrichment between pre-HH WT and post-HH Shh ${ }^{-/}$limbs (Figure S6C-E, Fisher's extract test $\mathrm{p}$-value= 0.14). Since Hand2 is only required for limb bud initiation (Galli et al., 2010), the absence of increased motif enrichment in pre- $\mathrm{HH}$ limb buds does not support a role for HAND2 in regulating this population 
of GLI3-bound early enhancers. We also note that $\mathrm{Tb} \times 2$ and $\mathrm{Tb} \times 3$ are not reduced in pre-HH Gli3 ${ }^{-/}$limb buds as would be expected if GLI3 had a significant role in co-activating these HAND2 target genes (Supplemental File 3).

\section{Discussion}

GLI repressors have been proposed to play a significant role in 'pre-patterning' the anteriorposterior limb bud prior to the onset of $\mathrm{HH}$ signaling (Chiang et al., 2001; Litingtung et al., 2002; Osterwalder et al., 2014; Panman and Zeller, 2003; te Welscher et al., 2002; Zhulyn et al., 2014; Wyngaarden et al., 2011; reviewed in Zuniga and Zeller, 2020). Unexpectedly, we find that GLI3 does not act as a transcriptional repressor in the early limb bud before the onset of $\mathrm{HH}$ signaling. Although the GLI3-R isoform is produced at comparable levels and binds to chromatin, it does not mediate deacetylation of H3K27ac or chromatin compaction at enhancers, as it does after $\mathrm{HH}$ signaling (Lex et al., 2020). Moreover, there is little to no upregulated gene expression in early $\mathrm{Gli3}^{-/}$limb buds. The lack of GLI repressor activity prior to the onset of Hedgehog signaling is inconsistent with the current pre-patterning model, instead it suggests that the repressive patterning activities of GLI3 in the limb bud are initiated concurrently with, or after, the initiation of $\mathrm{HH}$ signaling (Figure 6). This suggests that the widespread alterations in gene expression present in ciliopathies are primarily caused by misexpression of normally repressed GLI3 target genes during ongoing Hedgehog signaling rather than by precocious de-repression.

\section{GLI3-R is transcriptionally inert in early limb buds}

The absence of GLI repression in the early limb bud could be caused by inert GLI-R isoforms or by target genes that are not yet competent for GLI repression (Figure $4 \mathrm{I}, \mathrm{J}$ ). Our results are 
consistent with the co-occurrence of both of these mechanisms. GLI3 does not bind to almost a third of all HH-responsive GBRs that will be bound at E10.5, and most unbound regions having not yet acquired a poised conformation (Figure 1; Figure $\mathrm{S} 1 \mathrm{G}, \mathrm{H}$ ). In this case, pioneer factors may be required to prime this subset of GBRs before GLI3 can regulate them, as has been proposed for SOX2's role in activating GLI neural enhancers (Oosterveen et al., 2012; Peterson et al., 2012). The presence of the polycomb repressive mark, H3K27me3, at the promoters of many predicted $\mathrm{HH}$ target genes, provides an additional mechanism of GLI3-independent repression, as it is highly enriched at many promoters in E9.25 limbs, but is greatly reduced or absent from post-HH limb buds (Figures 4F-J, S4). Notably, GLI3 de-repression is unlikely to mediate this reduction, as many of these genes also lack H3K27me3 in E10.5 Shh ${ }^{-/}$limb buds (Lex et al., 2020). This suggests that removal of $\mathrm{H} 3 \mathrm{~K} 27 \mathrm{me} 3$ is largely independent of $\mathrm{HH}$ signaling and GLI3 regulation, despite reports demonstrating that $\mathrm{H} 3 \mathrm{~K} 27$ me3 enrichment at some $\mathrm{HH}$ targets is resolved in a HH-dependent manner (Figures 4F-J, S4)(Shi et al., 2014). The H3K27me3 clearing at these genes is most likely mediated by the loss of unknown, GLIindependent transcriptional repressors as has been proposed within the developing brain (He et al., 2020).

Unlike the previous examples, the class of genes that are bound by GLI3 and not enriched for H3K27me3 are more likely to be competent for GLI repression in pre-HH limb buds (Figure 4J). However, these genes are also not upregulated in Gli3 ${ }^{-}$limb buds (Fig. 4B,D), suggesting an absence of GLI3 repressor function at this stage. Several additional lines of evidence support this suggestion. First, Hand2 is broadly expressed in the anterior domain of pre-HH limb buds where there is co-localization with GLI3-R that persists through E9.5 (Figure 4D,E; Osterwalder et al., 2014). Second, H3K27ac levels at $\mathrm{HH}$ responsive GBRs do not increase in pre-HH Gli3 ${ }^{-/}$ limb buds (Figure 3) as they do in post-HH Gli3 $\%$ limb buds, even at genes that appear competent for GLI-repression (Figure 3B,E; 4J; Lex et al., 2020). Third, while most HH- 
responsive enhancers have lower $\mathrm{H} 3 \mathrm{~K} 27 \mathrm{ac}$ enrichment in pre- $\mathrm{HH}$ limb buds than in post-HH limb buds (Figure 2B,C), exceptional enhancers with higher levels of H3K27ac do not further increase acetylation levels in Gli3 ${ }^{-/}$limb buds, excluding the possibility that lack of increased H3K27ac levels are due to lack of a HH-independent activator (Figure S2B-D). Finally, HHresponsive GBRs are broadly accessible at pre-HH stages and up until $\sim 10$ hours after the time of $\mathrm{HH}$ induction, in contrast to the loss of accessibility seen under conditions of maximal GLI repression in post-HH E10.5 limbs (Figure 5).

\section{Mechanisms for establishing GLI-repressor activity}

One mechanism for regulating repressor activity is the variable expression of required co-factors as exemplified by the Brinker repressor (Upadhyai and Campbell, 2013). Consistent with this scenario, the genes encoding several co-repressors implicated with GLI repression in various contexts, including Ski, Smarcc1 and Atrophin1 (Dai et al., 2002; Jeon and Seong, 2016; Zhang et al., 2013) are significantly downregulated prior to $\mathrm{HH}$ induction compared to at E10.5

(Supplemental File 3). Conversely, a protein enriched in early limb buds could inhibit GLI3 repressor activity, as has been shown for HOXD12 in post-HH limb buds (Chen et al., 2004). Although HDACs are enriched at GBRs in both pre- and post-HH limb buds (Figure 2E,G), the lack of increased $\mathrm{H} 3 \mathrm{~K} 27 \mathrm{ac}$ enrichment in $\mathrm{Gli}^{-/-}$limb buds suggests that they are either inactive or not regulated by GLI3. Interestingly, both HDAC1 and HDAC2 initially co-localize at many GBRs at E9.25, while in post-HH limb buds, most remain bound by HDAC1 but lose HDAC2 enrichment. In other contexts, HDAC2 is required to recruit, but not maintain HDAC1 at some enhancers (Somanath et al. 2017), and a similar mechanism could enable the assembly of an HDAC1-containing GLI repression complex. As HDACs require co-repressor complexes to guide them to their substrates (reviewed in Adams et al., 2018), the absence of a functional GLI3 co-repressor might prevent them from being regulated by HDACs. Alternatively, rather 
than missing a co-repressor, it is possible that GLI3-R lacks additional, uncharacterized posttranslational modifications required for its activity, or to functionally interact with its repression complex (Figure 6D).

\section{Temporal onset of GLI transcriptional repression}

GLI-dependent restriction of chromatin accessibility can first be detected by ATAC-seq at 28$30 \mathrm{~S}$ when a few $\mathrm{HH}$-responsive GBRs have significantly reduced accessibility in $\mathrm{Sh}^{-/}$limb buds. However, most regions are actually more accessible than in E10.5 Shh ${ }^{-/}$, suggesting that GLI repression is not widespread at this time (Figure 5). Focusing on Hand2 as a likely direct target of GLI3 repression (Osterwalder et al., 2014; Vokes et al., 2008; te Welscher et al., 2002), there is no reduction in chromatin accessibility at this locus in E10 Shh ${ }^{-/}$limbs as is seen at E10.5 (Figure 5A). Hand2 expression does not become posteriorly restricted until around 26S (Figure 4E), an observation that is consistent with previous findings (Osterwalder et al., 2014), suggesting that GLI3 transcriptional repression of Hand2 commences around 26S, a timepoint coinciding with the activation of Shh expression in the limb bud (Figure 1A,B, Figure S1 A,B) (Charite et al., 2000; Zhu et al., 2008). The lack of detectable reductions in chromatin accessibility at a time shortly after repression of Hand2 has initiated, indicates that GLIdependent compaction likely occurs as a later step in GLI3-mediated repression.

The onset of GLI transcriptional repression is accompanied by a re-distribution of ciliary GLI3 into the axoneme, suggestive of a possible increase in GLI3 trafficking that coincides with the time when GLI3 starts to functionally repress target genes. We propose that increased GLI3 trafficking is facilitated by developmental changes to unknown ciliary processing components that regulate GLI3-R activity (Figure 6D). Since there are comparable levels of GLI3-R protein present within the pre- and post-HH limb buds (Fig. 1C), this regulation seems most likely to affect an unknown post-translational modification to GLI3-R rather than the processing of GLI3- 
FL into truncated GLI3-R (Figure 6D). If this is the case, then the activation of GLI repression is dependent on the status of the cilia and GLI transcriptional repression may be dynamically regulated in different developmental contexts.

\section{A Model for establishing GLI3-mediated repression}

Our findings provide genetic and genomic evidence that GLI3-R is inert prior to $\mathrm{HH}$ induction in the limb bud. As GLI3-dependent chromatin compaction is established in E10.5, but not E10 $S h h^{-/}$limb buds, it also suggests that GLI repression is likely established temporally during limb development through $\mathrm{HH}$-independent mechanisms. Rather than preventing initial expression of $\mathrm{HH}$ targets, most genes repressed by GLI3 in later limb development are initially expressed in pre-HH limb buds (Figure 6A; Supplemental File 3). Once repression is later established through unknown mechanisms, GLI3 functions to restrict target gene expression to the posterior limb (Figure 6B-D). More broadly, this work indicates that GLI repression is not a default state and instead must be acquired in context-dependent fashion. This has major implications for our understanding of the pathway itself and whether genes and enhancers must gain competency to be regulated by $\mathrm{HH}$ signaling. 


\section{Acknowledgements}

We thank Matt Anderson and Mark Lewandoski for sharing their protocol for clearing embryos, We thank the Henikoff Lab and EpiCypher for providing CUT\&Tag reagents, and Jonathan Eggenschwiler for providing the GLI2 antibody. We thank Jessica Podnar from the Genomic Sequencing and Analysis Facility at the University of Texas at Austin, Anna Webb from the Center for Biomedical Research Support at the University of Texas at Austin for technical advice, and Janani Ramachandran for comments on this manuscript. This work was supported by NIH R01HD073151 (to SAV and HJ), F31DE027597 (to RKL) and R01HG009518 (to HJ).

\section{Author Contributions}

Conceptualization, R.K.L. and S.A.V.; Methodology, R.K.L, W.Z., Z.J., Software J.D.K.;

Validation K.E.W.; Formal Analysis, R.K.L., W.Z., Z.J.; Investigation, R.K.L., W.Z., Z.J., K.N.F.,

K.E.S., K.E.W.; Data curation, R.K.L., W.Z.; Writing - Original Draft, R.K.L. and S.A.V.; Writing

- Review \& Editing, S.A.V, R.K.L, W.Z., H.J.; Supervision, S.A.V., H.J.; Funding Acquisition, S.A.V., H.J., R.K.L.

\section{Declaration of Interests}

The authors declare no competing interests 


\section{STAR Methods}

\section{KEY RESOURCES TABLE}

\begin{tabular}{|c|c|c|}
\hline REAGENT or RESOURCE & SOURCE & IDENTIFIER \\
\hline \multicolumn{3}{|l|}{ Antibodies } \\
\hline $\begin{array}{l}\text { Anti-HDAC1 } \\
\text { (rabbit polyclonal) }\end{array}$ & Abcam & $\begin{array}{l}\text { Abcam Cat \#ab7028 } \\
\text { RRID:AB_305705 }\end{array}$ \\
\hline $\begin{array}{l}\text { Anti-HDAC2 } \\
\text { (rabbit polyclonal) }\end{array}$ & Abcam & $\begin{array}{l}\text { Abcam Cat \#ab7029 } \\
\text { RRID:AB_305706 }\end{array}$ \\
\hline Anti-H3K27ac (rabbit polyclonal) & Abcam & $\begin{array}{l}\text { Abcam Cat\# ab4729, } \\
\text { RRID:AB_2118291 }\end{array}$ \\
\hline Anti-H3K27me3 (rabbit polyclonal) & Abcam & $\begin{array}{l}\text { Abcam Cat\# Ab195477, } \\
\text { RRID:AB_2819023 }\end{array}$ \\
\hline Anti-H3K4me1 (rabbit polyclonal) & Abcam & $\begin{array}{l}\text { Abcam Cat\# Ab8895, } \\
\text { RRID:AB_306847 }\end{array}$ \\
\hline Anti-H3K4me2 (rabbit polyclonal) & Millipore & $\begin{array}{l}\text { Millipore Cat\# 07-030, } \\
\text { RRID:AB_11213050 }\end{array}$ \\
\hline Anti-M2 FLAG (mouse monoclonal) & Sigma & $\begin{array}{l}\text { Sigma-Aldrich Cat\# } \\
\text { F3165, RRID:AB_259529 }\end{array}$ \\
\hline Anti-B-actin (rabbit polyclonal) & $\begin{array}{l}\text { Cell Signaling } \\
\text { Technology }\end{array}$ & $\begin{array}{l}\text { Cell Signaling } \\
\text { Technology Cat\# } 8457 \text {, } \\
\text { RRID:AB_10950489 }\end{array}$ \\
\hline Donkey-anti-mouse & $\begin{array}{l}\text { Jackson Immuno- } \\
\text { Research }\end{array}$ & $\begin{array}{l}\text { Jackson } \\
\text { ImmunoResearch Labs } \\
\text { Cat\# 715-035-150, } \\
\text { RRID:AB_2340770 }\end{array}$ \\
\hline Donkey-anti-rabbit & $\begin{array}{l}\text { Jackson Immuno- } \\
\text { Research }\end{array}$ & $\begin{array}{l}\text { Jackson } \\
\text { ImmunoResearch Labs } \\
\text { Cat\# 711-005-152, } \\
\text { RRID:AB_2340585 }\end{array}$ \\
\hline Dynabeads M-280 Sheep Anti-Rabbit IgG & $\begin{array}{l}\text { Invitrogen, } \\
\text { Thermo Fisher } \\
\text { Scientific }\end{array}$ & $\begin{array}{l}\text { Thermo Fisher Scientific } \\
\text { Cat\# 11203D, } \\
\text { RRID:AB_2783009 }\end{array}$ \\
\hline Anti-ARL13b (rabbit polyclonal) & Proteintech & $\begin{array}{l}\text { Proteintech Cat\#17711- } \\
\text { 1-AP } \\
\text { RRID:AB_2060867 }\end{array}$ \\
\hline Anti-GLI2 (guinea pig) & $\begin{array}{l}\text { Gift from Dr. } \\
\text { Jonathon } \\
\text { Eggenschwiler }\end{array}$ & \\
\hline Anti-Gamma Tubulin (mouse monoclonal) & & $\begin{array}{l}\text { Sigma \#T6557 } \\
\text { RRID:AB_477584 }\end{array}$ \\
\hline
\end{tabular}




\begin{tabular}{|c|c|c|}
\hline $\begin{array}{l}\text { Alexa488 } \\
\text { (Goat anti-Rabbit) }\end{array}$ & Thermofisher & $\begin{array}{l}\text { Thermofisher } \\
\text { Cat\#A32731 } \\
\text { RRID:AB_2633280 }\end{array}$ \\
\hline $\begin{array}{l}\text { Alexa568 } \\
\text { (Goat anti-Mouse) }\end{array}$ & Thermofisher & $\begin{array}{l}\text { Thermofisher } \\
\text { Cat\#A11004 } \\
\text { RRID:AB_2534072 }\end{array}$ \\
\hline $\begin{array}{l}\text { Alexa594 } \\
\text { (Donkey anti-Guinea Pig) }\end{array}$ & $\begin{array}{l}\text { Jackson } \\
\text { ImmunoResearch }\end{array}$ & $\begin{array}{l}\text { Jackson } \\
\text { ImmunoResearch } \\
\text { Cat\#706-585-148 } \\
\text { RRID:AB_2340474 }\end{array}$ \\
\hline \multicolumn{3}{|l|}{ Bacterial and virus strains } \\
\hline \multicolumn{3}{|l|}{ Biological samples } \\
\hline \multicolumn{3}{|l|}{ Chemicals, peptides, and recombinant proteins } \\
\hline CUTANA pAG-MNase & EpiCypher & EpiCypher Cat \#15-1016 \\
\hline Protein A-Tn5 (pA-Tn5) fusion protein & $\begin{array}{l}\text { Henikoff Lab, Fred } \\
\text { Hutch }\end{array}$ & \\
\hline Liberase & Roche & $\begin{array}{l}\text { Roche Cat\# } \\
05401119001\end{array}$ \\
\hline Concanavalin A (ConA)-coated magnetic beads & Bangs Laboratories & $\begin{array}{l}\text { Bangs Laboratories Cat\# } \\
\text { BP531 }\end{array}$ \\
\hline \multicolumn{3}{|l|}{ Critical commercial assays } \\
\hline NEBNext High-Fidelity 2x PCR Master Mix & New England Biolabs & NEB Cat\# M0541 \\
\hline NEBNext Ultra II DNA Library Prep Kit for Illumina & New England Biolabs & NEB Cat\#E7645S \\
\hline SparQ PureMag Beads & Quantabio & Quantabio Cat \#95196 \\
\hline NEBNext Multiplex Oligos for Illumina (96 Index primers) & New England Biolabs & NEB Cat \# E6609S \\
\hline Amersham ECL Prime & GE Healthcare & \#GERPN2232 \\
\hline HCR v3.0 Probe sets, amplifiers and buffers & Molecular Instruments & \\
\hline \multicolumn{3}{|l|}{ Deposited data } \\
\hline CUT\&RUN, CUT\&Tag, ChIP-seq, ATAC sequencing & $\begin{array}{l}\text { Gene Expression } \\
\text { Omnibus (GEO) }\end{array}$ & TBD \\
\hline 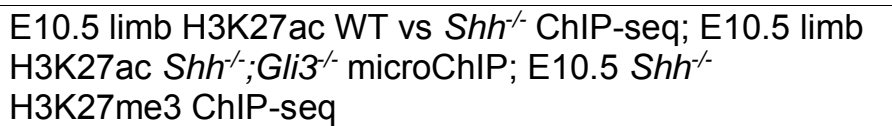 & $\begin{array}{l}\text { Gene Expression } \\
\text { Omnibus (GEO) }\end{array}$ & GSE108880 \\
\hline Mouse reference genome NCBI build GRCm38/mm10 & $\begin{array}{l}\text { Gene Expression } \\
\text { Omnibus (GEO) }\end{array}$ & \\
\hline \multicolumn{3}{|l|}{ Experimental models: Cell lines } \\
\hline Experimental models: Organisms/strains & & \\
\hline
\end{tabular}




\begin{tabular}{|c|c|c|}
\hline M. musculus: Gli3 Xt-J Gli3 & Jackson Laboratory & $\begin{array}{l}\text { Jackson Cat\# 000026, } \\
\text { MGI Cat\# 2169581, } \\
\text { RRID:MGI:2169581 }\end{array}$ \\
\hline M. musculus: Shhtm1amc Shht/- & Jackson Laboratory & $\begin{array}{l}\text { Jackson Cat\# 003318, } \\
\text { MGI Cat\# 3584154, } \\
\text { RRID:MGI:3584154 }\end{array}$ \\
\hline M. musculus: Gli3 FLAG & $\begin{array}{l}\text { Laboratory of Dr. Andy } \\
\text { McMahon }\end{array}$ & \\
\hline $\begin{array}{l}\text { M. musculus: Swiss Webster } \\
\text { Wildtype }\end{array}$ & Charles River & $\begin{array}{l}\text { Charles River Cat\# NCl } \\
551 \\
\text { IMSR Cat\# TAC:sw, } \\
\text { RRID:IMSR_TAC:sw }\end{array}$ \\
\hline \multicolumn{3}{|l|}{ Oligonucleotides } \\
\hline \multicolumn{3}{|l|}{ Recombinant DNA } \\
\hline \multicolumn{3}{|l|}{ Software and algorithms } \\
\hline limma & (Ritchie, et al., 2015) & $\begin{array}{l}\text { LIMMA, } \\
\text { RRID:SCR_010943 }\end{array}$ \\
\hline R statistical software & (Team, 2011) & $\begin{array}{l}\text { R Project for Statistical } \\
\text { Computing, } \\
\text { RRID:SCR_001905 }\end{array}$ \\
\hline MACS version 2.1.0 & $\begin{array}{l}\text { (Zhang, et al., 2008) } \\
\text { https://github.com/taoli } \\
\text { u/MACS }\end{array}$ & $\begin{array}{l}\text { MACS, } \\
\text { RRID:SCR_013291 }\end{array}$ \\
\hline CisGenome & (Ji, et al., 2008) & $\begin{array}{l}\text { CisGenome, } \\
\text { RRID:SCR_001558 }\end{array}$ \\
\hline JASPAR motif database & (Khan, et al., 2018) & $\begin{array}{l}\text { JASPAR, } \\
\text { RRID:SCR_003030 }\end{array}$ \\
\hline Transfac motif database & (Matys, et al., 2006) & $\begin{array}{l}\text { TRANSFAC, } \\
\text { RRID:SCR_005620 }\end{array}$ \\
\hline Other & & \\
\hline
\end{tabular}




\section{Genomic Datasets}

CUT\&RUN, CUT\&Tag, ATAC-Seq, ChIP-seq and RNA-seq datasets were deposited in GEO (GSE178838). The H3K27ac, H3K4me2 (GSE108880) and H3K4me1 ChIP-seq (GSE86690) datasets used in this study were processed and analyzed as all other ChIP experiments were done, described above. All chromosomal coordinates refer to the $\mathrm{mm} 10$ version of the mouse genome.

\section{Embryonic manipulations}

Experiments involving mice were approved by the Institutional Animal Care and Use Committee at the University of Texas at Austin (protocol AUP-2019-00233). The Gli3 ${ }^{X t-J}$ and Shhtmimc null alleles (Hui and Joyner 1993; Dassule et al. 2000) were maintained on a Swiss Webster background. The Gli3 ${ }^{3 X F A G}$ allele, with an N-terminal 3XFLAG-epitope, (Lopez-Rios et al. 2014; Lorberbaum et al. 2016) was maintained on a mixed background. For all genomic experiments, with the exception of $\mathrm{Shh}^{-/}$; Gli3/- ATAC samples which had to be individually genotypes, fresh E9.25 (21-23S) and E10.5 (32-35S) forelimb buds were pooled from multiple litters of the specified genotype. For ATAC-seq experiments with WT forelimbs (28-30S; 35S), and Shh ${ }^{-1}$ forelimbs (28-30S; 35S), posterior halves of forelimbs from 2-3 embryos were dissected and pooled. For RNA-seq, E9.25 whole forelimbs or E10.5 anterior halves forelimbs of WT and Gli3 /-forelimbs were pooled from 3-4 embryos; 3-4 biological replicates were used for each stage and genotype.

\section{In situ hybridization}

In situ hybridization done as previously described (Lewandowski et al., 2015). Fluorescent in situ hybridization was performed using HCR v3.0 Molecular Instruments reagents and was performed according to manufacturer's protocol (Choi et al., 2018) except that probes were incubated overnight with a concentration of 16nM. Embryos were embedded in Ultralow gelling 
temperature agarose (Sigma A5030), cleared in Ce3D++ (Anderson et al., 2020) and visualized on a Zeiss LSM 710 confocal microscope and are shown as maximal intensity z-stack projections.

\section{Immunofluorescence}

Embryos were sucrose protected ( $30 \%$ sucrose) at RT and embedded in OCT. $12 \mu \mathrm{m}$ cryosections were immediately fixed in $100 \%$ methanol for 3 minutes at $-18 \mathrm{C}$. Subsequently, sections were permeabilized in $0.1 \%$ PBST at RT for 15 min., blocked in 3\% BSA (Fisher Scientific; BP1600-100), 5\% Normal Goat Serum (Jackson Immunoresearch) in 0.1\% PBST for $1 \mathrm{hr}$. at RT. Primary antibodies were incubated overnight with: 1:500 M2 FLAG (Sigma-Aldrich; F3165) and 1:500 Arl13b (Proteintech; 17711-1-AP). Secondary antibodies were incubated in block at RT for $1 \mathrm{hr}$. at the following dilutions: 1:500 Alexa568 (Thermo Fisher Scientific; A11004) and 1:500 Alexa488 (Thermo Fisher Scientific; A-11034) and mounted with Prolong Gold mounting media (Thermo Scientific; P36930) prior to imaging.

\section{Western Blots}

Western blots were incubated for 1 hour at room temperature in 3\% milk: 1:4000 M2 Flag (Sigma \#F3165) and 1:2000 B-actin (Cell Signaling \#8457). Secondary antibodies were incubated for 1 hours at room temperature in 3\% milk: 1:5000 Donkey anti-mouse (Jackson \#715-035-150), Donkey anti-rabbit (Jackson \#711-005-0152). Blots were developed using Amersham ECL Prime (GE Healthcare \#GERPN2232).

\section{Chromatin Immunoprecipitation}

ChIP-Seq was performed on whole E9.25 (21-23S) forelimbs pooled from 30-40 embryos as previously described (Vokes et al. 2008) with the following modifications. Cells were dissociated 
with $100 \mu \mathrm{g} / \mathrm{ml}$ Liberase (Roche 05401119001$)$ and fixed for 15 minutes in $1 \%$ formaldehyde.

After cell lysis, chromatin was sheared in buffer containing $0.25 \%$ SDS with a Covaris S2

focused ultrasonicator. Antibodies used for ChIP experiments include H3K27ac (Abcam \#ab4729) and H3K4me2 (Millipore \#07-030). Libraries were generated using the NEBNext Ultra II library preparation kit with 15 cycles of PCR amplification (NEB E7645) and sequenced to a depth of $>40$ million reads per sample, using two biological replicates. Peaks were called using CisGenome version 2.1.0 (Ji et al. 2008). The read numbers were adjusted by library size and $\log 2$ transformed after adding a pseudo-count of 1 . The differential analyses were performed using limma (Ritchie et al. 2015); peaks with an FDR $<0.05$ were considered to have differential enrichment.

\section{ATAC-Seq}

WT E9.25 (21-23S) forelimbs or posterior forelimbs from E10 (28-30S) or E10.5 (35S) forelimbs were dissected and pooled from WT embryos $(n=3, n=3)$. Posterior forelimbs from E10 (28-30S) or E10.5 (35S) were pooled from Shh ${ }^{--}$embryos $(n=3, n=3)$. ATAC used components from the Nextera DNA Library Preparation Kit (Illumina) as described previously (Buenrostro et al. 2015) with the following variations. Limb buds were dissociated in $100 \mu \mathrm{g} / \mathrm{ml}$ Liberase (Roche 05401119001) and lysed for $10 \mathrm{~min}$. at $4^{\circ} \mathrm{C}$, rotating, in a nuclear permeabilization buffer $(5 \%$ BSA, $0.2 \%$ NP-40, $1 \mathrm{mM} \mathrm{DTT}$ in $\mathrm{Ca}+/ \mathrm{Mg}+$ free PBS + protease inhibitor) and then centrifuged for $10 \mathrm{~min}$. at $300 \mathrm{~g}, 4 \mathrm{C}$. Nuclei were resuspended, counted using Trypan Blue, and 30,000 nuclei from each WT and Shh/- sample were put into each transposase reaction. The transposase reaction (30uL volume) was carried out for 1 hour at $37^{\circ} \mathrm{C}$ with gentle agitation. Libraries were generated using 11 cycles of PCR amplification with NEB high fidelity 2x master mix (New England Biolabs), cleaned up with SparQ PureMag beads (QuantaBio) and sequenced on an Illumina NovaSeq using PE150 or SR100 to a depth of at least 40 million 
reads. Peaks were called using MACS2 with a fixed window size of 200bp and a q-value cutoff of 0.05 . Read counts from the peak regions were obtained and differential analysis was performed using DESeq2 (Love et al., 2014). Peaks with FDR $<0.05$ in the differential test were considered to have significant chromatin accessibility difference.

Footprinting analysis was performed using HINT (Li et al., 2019) based on the ATAC-seq data. After obtaining the DNA footprints, GLI3 and HAND2 motifs were mapped to the footprints which overlap with regions that have pre-HH only GLI3 binding, E10.5 HAND2 binding, and E9.25 H3K27ac signals (Figure S6C) using CisGenome (Ji et al., 2008). Motif enrichment (Figure S6E) is calculated as the ratio between the percentage of footprints containing the motif in the E9.25 WT samples and that in the E10.5 Sh/ ${ }^{-/}$samples. Fisher's extract test is applied to test if the motif enrichment is different between E9.25 WT and E10.5 Shh ${ }^{-/}$(GLI3 p-value $=0.87$, HAND2 p-value $=0.14)$

\section{CUT\&Tag}

Experiments were performed as described in (Kaya-Okur et al., 2019) with the following modifications. 100,000 cells from E9.25 (21-23S) forelimbs were dissociated, bound to Concanavalin beads and incubated overnight at $4^{\circ} \mathrm{C}$ on a nutator with primary antibodies. Antibodies were used at the following concentrations: $1 \mu \mathrm{g} / \mathrm{mL}$ of H3K4me1 (Abcam \#ab8895) and $5 \mu \mathrm{g} / \mathrm{mL}$ of H3K27me3 (abcam \#ab195477). The following day, samples were incubated at room temperature for $30 \mathrm{~min}$. with secondary antibody, 1:500 Donkey anti-rabbit (Jackson ImmunoResearch \#711-005-152). CUT\&Tag transposases used were gifted from the Henikoff lab and EpiCyphr (now commercially available). Libraries were generated using NEB high fidelity 2x master mix with 14 PCR cycles and cleaned up to remove adapters using or SparQ PureMag beads (QuantaBio). Samples were sequenced on an Illumina NextSeq 500 instrument 
using PEx75 to a depth of depth of 3-5 million reads. Peaks were called using SEACR (Meers et al., 2019).

\section{CUT\&RUN}

Experiments were performed according to EpiCypher's CUTANA CUT\&RUN protocol (EpiCypher \#15-1016) with the following modifications. 200,000-300,000 cells from E9.25 (2123S) forelimbs and anterior E10.5 (32-35S) forelimbs were incubated overnight at $4^{\circ} \mathrm{C}$ on a nutator with 1:250 FLAG primary antibody (Sigma \#F3165), 1:100 HDAC1 (Abcam ab7028) or 1:200 HDAC2 (Abcam ab7029). The following day, samples were incubated at room temperature for $30 \mathrm{~min}$. with secondary antibody, 1:500 Donkey anti-mouse (Jackson ImmunoResearch \#715-035-150) or Donkey anti-rabbit (Jackson ImmunoResearch \#711-005152), followed by three washes in Digitonin wash buffer. CUTANA pAG-MNase was then incubated with samples for 10 minutes at room temperature and then the MNase reaction was performed for 2 hours at $4^{\circ} \mathrm{C}$ on a nutator. Libraries were generated using NEBNext Ultra II DNA Library Prep Kit with 14 PCR cycles and cleaned up to remove adapters using AMPure XP beads (Beckman Coulter) or SparQ PureMag beads (QuantaBio). Samples were sequenced on an Illumina NextSeq 500 instrument using PEx75 to a depth of depth of 3-5 million reads. Peaks were called using MACS (Zhang et al., 2008).

\section{Intron retention rates}

The formula (1-N)/M was used to calculate the intron retention rate for each gene, where $\mathrm{N}=$ the number of reads overlapping with any of the gene's exons and $M=$ the number of reads overlapping with the body of the gene. Limma (Ritchie et al., 2015) was used to identify differential intron retention rate between E9.25 and E10.5 Gli3\% limbs, FDR cutoff $<0.05$. 


\section{Identification of GLI target genes}

To identify GLI target genes, we developed a weighted system which incorporated HHresponsive GBRs, poised chromatin modifications, active promoter marks and genes identified as being significantly downregulated in E10.5 Sh/ ${ }^{-/}$RNA-seq compared to WT limb buds (FDR=0.05; WT/Shh ${ }^{-/}$FC <0) (Lewandowski et al., 2015; GSE58222). HH-responsive GBRs were intersected with poised enhancer modifications from H3K4me1 ChIP-seq (GSE86690), H3K4me2 ChIP-seq and E10.5 ATAC-seq datasets (Lex et al., 2020; GSE108880) from E10.5 WT limb buds. HH-responsive GBRs enriched for poised enhancers modifications, and thus increasing the probably of being active enhancers, were given a higher weight than ones without modifications. Genes identified as downregulated in Shh mutant limbs were intersected with H3K4me3 (GSE86698) and H3K4me6 (GSE8669) E10.5 WT limb datasets. Genes enriched for these active promoter marks were given more weight. The weighted HH-responsive GBRs and weighted genes were then intersected based on proximity, up to $500 \mathrm{~kb}$, and within the same topologically associated domain (TAD) (GSE96107). Genes were identified as a putative $\mathrm{HH}$ targets based on their proximity to $\mathrm{HH}$-responsive GBRs and the number of $\mathrm{HH}$ responsive GBRs, such that a gene with multiple HH-responsive GBRs, enriched for poised enhancer marks, would rank higher than a gene with HH-responsive GBRs found more distally. https://github.com/Boksunni/Predicted-HH-List-Generation 


\section{References}

Adams, G.E., Chandru, A., and Cowley, S.M. (2018). Co-repressor, co-activator and general transcription factor: the many faces of the Sin3 histone deacetylase (HDAC) complex. Biochem J 475, 3921-3932.

Anderson, M.J., Magidson, V., Kageyama, R., and Lewandoski, M. (2020). Fgf4 maintains Hes7 levels critical for normal somite segmentation clock function. Elife 9.

Aza-Blanc, P., Ramírez-Weber, F.A., Laget, M.P., Schwartz, C., and Kornberg, T.B. (1997). Proteolysis that is inhibited by hedgehog targets Cubitus interruptus protein to the nucleus and converts it to a repressor. Cell 89, 1043-1053.

Balaskas, N., Ribeiro, A., Panovska, J., Dessaud, E., Sasai, N., Page, K.M., Briscoe, J., and Ribes, V. (2012). Gene regulatory logic for reading the Sonic Hedgehog signaling gradient in the vertebrate neural tube. Cell 148, 273-284.

Bénazet, J.-D., and Zeller, R. (2009). Vertebrate Limb Development: Moving from Classical Morphogen Gradients to an Integrated 4-Dimensional Patterning System. Cold Spring Harb Perspect Biol 1, a001339.

Charite, J., McFadden, D.G., and Olson, E.N. (2000). The bHLH transcription factor dHAND controls Sonic hedgehog expression and establishment of the zone of polarizing activity during limb development. Development 127, 2461-2470.

Chen, Y., Knezevic, V., Ervin, V., Hutson, R., Ward, Y., and Mackem, S. (2004). Direct interaction with Hoxd proteins reverses Gli3-repressor function to promote digit formation downstream of Shh. Development 131, 2339-2347.

Chiang, C., Litingtung, Y., Harris, M.P., Simandl, B.K., Li, Y., Beachy, P.A., and Fallon, J.F. (2001). Manifestation of the Limb Prepattern: Limb Development in the Absence of Sonic Hedgehog Function. Developmental Biology 236, 421-435.

Choi, H.M.T., Schwarzkopf, M., Fornace, M.E., Acharya, A., Artavanis, G., Stegmaier, J., Cunha, A., and Pierce, N.A. (2018). Third-generation in situ hybridization chain reaction: multiplexed, quantitative, sensitive, versatile, robust. Development 145.

Dai, P., Shinagawa, T., Nomura, T., Harada, J., Kaul, S.C., Wadhwa, R., Khan, M.M., Akimaru, H., Sasaki, H., Colmenares, C., et al. (2002). Ski is involved in transcriptional regulation by the repressor and full-length forms of Gli3. Genes Dev. 16, 2843-2848.

Elliott, K.H., Chen, X., Salomone, J., Chaturvedi, P., Schultz, P.A., Balchand, S.K., Servetas, J.D., Zuniga, A., Zeller, R., Gebelein, B., et al. (2020). Gli3 utilizes Hand2 to synergistically regulate tissue-specific transcriptional networks. ELife 9, e56450.

Ernst, J., Kheradpour, P., Mikkelsen, T.S., Shoresh, N., Ward, L.D., Epstein, C.B., Zhang, X., Wang, L., Issner, R., Coyne, M., et al. (2011). Mapping and analysis of chromatin state dynamics in nine human cell types. Nature 473, 43-49. 
Fernandez-Teran, M., Piedra, M.E., Kathiriya, I.S., Srivastava, D., Rodriguez-Rey, J.C., and Ros, M.A. (2000). Role of dHAND in the anterior-posterior polarization of the limb bud: implications for the Sonic hedgehog pathway. Development 127, 2133-2142.

Galli, A., Robay, D., Osterwalder, M., Bao, X., Bénazet, J.-D., Tariq, M., Paro, R., Mackem, S., and Zeller, R. (2010). Distinct roles of Hand2 in initiating polarity and posterior Shh expression during the onset of mouse limb bud development. PLoS Genet 6, e1000901.

Gros, J., and Tabin, C.J. (2014). Vertebrate Limb Bud Formation Is Initiated by Localized Epithelial-to-Mesenchymal Transition. Science 343, 1253-1256.

Guzzetta, A., Koska, M., Rowton, M., Sullivan, K.R., Jacobs-Li, J., Kweon, J., Hidalgo, H., Eckart, H., Hoffmann, A.D., Back, R., et al. (2020). Hedgehog-FGF signaling axis patterns anterior mesoderm during gastrulation. PNAS 117, 15712-15723.

Haycraft, C.J., Banizs, B., Aydin-Son, Y., Zhang, Q., Michaud, E.J., and Yoder, B.K. (2005). Gli2 and Gli3 localize to cilia and require the intraflagellar transport protein polaris for processing and function. PLoS Genet 1, e53.

He, P., Williams, B.A., Trout, D., Marinov, G.K., Amrhein, H., Berghella, L., Goh, S.-T., Plajzer-Frick, I., Afzal, V., Pennacchio, L.A., et al. (2020). The changing mouse embryo transcriptome at whole tissue and single-cell resolution. Nature 583, 760-767.

Ho, E.K., and Stearns, T. (2021). Hedgehog signaling and the primary cilium: implications for spatial and temporal constraints on signaling. Development 148.

Hoffmann, A.D., Yang, X.H., Burnicka-Turek, O., Bosman, J.D., Ren, X., Steimle, J.D., Vokes, S.A., McMahon, A.P., Kalinichenko, V.V., and Moskowitz, I.P. (2014). Foxf genes integrate tbx5 and hedgehog pathways in the second heart field for cardiac septation. PLoS Genet 10, e1004604.

Jeon, S., and Seong, R.H. (2016). Anteroposterior Limb Skeletal Patterning Requires the Bifunctional Action of SWI/SNF Chromatin Remodeling Complex in Hedgehog Pathway. PLOS Genetics 12, e1005915.

Ji, H., Jiang, H., Ma, W., Johnson, D.S., Myers, R.M., and Wong, W.H. (2008). An integrated software system for analyzing ChIP-chip and ChIP-seq data. Nature Biotechnology 26, 1293-1300.

Kaya-Okur, H.S., Wu, S.J., Codomo, C.A., Pledger, E.S., Bryson, T.D., Henikoff, J.G., Ahmad, K., and Henikoff, S. (2019). CUT\&Tag for efficient epigenomic profiling of small samples and single cells. Nature Communications 10, 1930.

Kitazawa, T., Machlab, D., Joshi, O., Maiorano, N., Kohler, H., Ducret, S., Kessler, S., Gezelius, H., Soneson, C., Papasaikas, P., et al. (2021). A unique bipartite Polycomb signature regulates stimulus-response transcription during development. Nat Genet 53, 379-391. 
Lewandowski, J.P., Du, F., Zhang, S., Powell, M.B., Falkenstein, K.N., Ji, H., and Vokes, S.A. (2015). Spatiotemporal regulation of GLI target genes in the mammalian limb bud. Developmental Biology 406, 92-103.

Lewis, P.M., Dunn, M.P., McMahon, J.A., Logan, M., Martin, J.F., St-Jacques, B., and McMahon, A.P. (2001). Cholesterol modification of sonic hedgehog is required for longrange signaling activity and effective modulation of signaling by Ptc1. Cell 105, 599612.

Lex, R.K., Ji, Z., Falkenstein, K.N., Zhou, W., Henry, J.L., Ji, H., and Vokes, S.A. (2020). GLI transcriptional repression regulates tissue-specific enhancer activity in response to Hedgehog signaling. ELife 9, e50670.

Li, Q., Lewandowski, J.P., Powell, M.B., Norrie, J.L., Cho, S.H., and Vokes, S.A. (2014). A Gli silencer is required for robust repression of gremlin in the vertebrate limb bud. Development 141, 1906-1914.

Li, Z., Schulz, M.H., Look, T., Begemann, M., Zenke, M., and Costa, I.G. (2019). Identification of transcription factor binding sites using ATAC-seq. Genome Biology 20, 45.

Litingtung, Y., Dahn, R.D., Li, Y., Fallon, J.F., and Chiang, C. (2002). Shh and Gli3 are dispensable for limb skeleton formation but regulate digit number and identity. Nature 418, 979-983.

Lopez-Rios, J., Duchesne, A., Speziale, D., Andrey, G., Peterson, K.A., Germann, P., Ünal, E., Liu, J., Floriot, S., Barbey, S., et al. (2014). Attenuated sensing of SHH by Ptch1 underlies evolution of bovine limbs. Nature 511, 46-51.

Love, M.I., Huber, W., and Anders, S. (2014). Moderated estimation of fold change and dispersion for RNA-seq data with DESeq2. Genome Biology 15, 550.

Mahlapuu, M., Ormestad, M., Enerbäck, S., and Carlsson, P. (2001). The forkhead transcription factor Foxf1 is required for differentiation of extra-embryonic and lateral plate mesoderm. Development 128, 155-166.

Margueron, R., and Reinberg, D. (2011). The Polycomb complex PRC2 and its mark in life. Nature 469, 343-349.

McFadden, D.G., McAnally, J., Richardson, J.A., Charité, J., and Olson, E.N. (2002). Misexpression of dHAND induces ectopic digits in the developing limb bud in the absence of direct DNA binding. Development 129, 3077-3088.

Meers, M.P., Tenenbaum, D., and Henikoff, S. (2019). Peak calling by Sparse Enrichment Analysis for CUT\&RUN chromatin profiling. Epigenetics \& Chromatin 12, 42. 
Méthot, N., and Basler, K. (1999). Hedgehog controls limb development by regulating the activities of distinct transcriptional activator and repressor forms of Cubitus interruptus. Cell 96, 819-831.

Mirza, A.N., McKellar, S.A., Urman, N.M., Brown, A.S., Hollmig, T., Aasi, S.Z., and Oro, A.E. (2019). LAP2 Proteins Chaperone GLI1 Movement between the Lamina and Chromatin to Regulate Transcription. Cell 176, 198-212.e15.

Nasr, T., Holderbaum, A.M., Chaturvedi, P., Agarwal, K., Kinney, J.L., Daniels, K., Trisno, S.L., Ustiyan, V., Shannon, J.M., Wells, J.M., et al. (2020). Disruption of a hedgehog-foxf1-rspo2 signaling axis leads to tracheomalacia and a loss of sox9+ tracheal chondrocytes. Dis Model Mech.

Niewiadomski, P., Kong, J.H., Ahrends, R., Ma, Y., Humke, E.W., Khan, S., Teruel, M.N., Novitch, B.G., and Rohatgi, R. (2014). Gli protein activity is controlled by multi-site phosphorylation in vertebrate Hedgehog signaling. Cell Rep 6, 168-181.

Oosterveen, T., Kurdija, S., Alekseenko, Z., Uhde, C.W., Bergsland, M., Sandberg, M., Andersson, E., Dias, J.M., Muhr, J., and Ericson, J. (2012). Mechanistic Differences in the Transcriptional Interpretation of Local and Long-Range Shh Morphogen Signaling. Developmental Cell 23, 1006-1019.

Osterwalder, M., Speziale, D., Shoukry, M., Mohan, R., Ivanek, R., Kohler, M., Beisel, C., Wen, X., Scales, S.J., Christoffels, V.M., et al. (2014). HAND2 Targets Define a Network of Transcriptional Regulators that Compartmentalize the Early Limb Bud Mesenchyme. Dev Cell 31, 345-357.

Panman, L., and Zeller, R. (2003). Patterning the limb before and after SHH signalling. J Anat 202, 3-12.

Panman, L., Galli, A., Lagarde, N., Michos, O., Soete, G., Zuniga, A., and Zeller, R. (2006). Differential regulation of gene expression in the digit forming area of the mouse limb bud by SHH and gremlin 1/FGF-mediated epithelial-mesenchymal signalling. Development 133, 3419-3428.

Parker, D.S., White, M.A., Ramos, A.I., Cohen, B.A., and Barolo, S. (2011). The cisRegulatory Logic of Hedgehog Gradient Responses: Key Roles for Gli Binding Affinity, Competition, and Cooperativity. Sci. Signal. 4, ra38-ra38.

Pekowska, A., Benoukraf, T., Zacarias-Cabeza, J., Belhocine, M., Koch, F., Holota, H., Imbert, J., Andrau, J.-C., Ferrier, P., and Spicuglia, S. (2011). H3K4 tri-methylation provides an epigenetic signature of active enhancers. The EMBO Journal 30,41984210.

Peterson, K.A., Nishi, Y., Ma, W., Vedenko, A., Shokri, L., Zhang, X., McFarlane, M., Baizabal, J.-M., Junker, J.P., van Oudenaarden, A., et al. (2012). Neural-specific Sox2 input and differential Gli-binding affinity provide context and positional information in Shh-directed neural patterning. Genes Dev 26, 2802-2816. 
Probst, S., Kraemer, C., Demougin, P., Sheth, R., Martin, G.R., Shiratori, H., Hamada, H., Iber, D., Zeller, R., and Zuniga, A. (2011). SHH propagates distal limb bud development by enhancing CYP26B1-mediated retinoic acid clearance via AER-FGF signalling. Development 138, 1913-1923.

Ritchie, M.E., Phipson, B., Wu, D., Hu, Y., Law, C.W., Shi, W., and Smyth, G.K. (2015). limma powers differential expression analyses for RNA-sequencing and microarray studies. Nucleic Acids Res 43, e47-e47.

Sharova, L.V., Sharov, A.A., Nedorezov, T., Piao, Y., Shaik, N., and Ko, M.S.H. (2009). Database for mRNA Half-Life of 19977 Genes Obtained by DNA Microarray Analysis of Pluripotent and Differentiating Mouse Embryonic Stem Cells. DNA Res 16, 45-58.

Shi, X., Zhang, Z., Zhan, X., Cao, M., Satoh, T., Akira, S., Shpargel, K., Magnuson, T., Li, Q., Wang, R., et al. (2014). An epigenetic switch induced by Shh signalling regulates gene activation during development and medulloblastoma growth. Nature Communications 5, 5425.

Skene, P.J., and Henikoff, S. (2017). An efficient targeted nuclease strategy for highresolution mapping of DNA binding sites. ELife 6 , e21856.

Somanath, P., Klein, R.H., and Knoepfler, P.S. (2017). CRISPR-mediated HDAC2 disruption identifies two distinct classes of target genes in human cells. PLOS ONE 12, e0185627.

Upadhyai, P., and Campbell, G. (2013). Brinker possesses multiple mechanisms for repression because its primary co-repressor, Groucho, may be unavailable in some cell types. Development 140, 4256-4265.

Vokes, S.A., Ji, H., Wong, W.H., and McMahon, A.P. (2008). A genome-scale analysis of the cis-regulatory circuitry underlying sonic hedgehog-mediated patterning of the mammalian limb. Genes Dev. 22, 2651-2663.

Wang, B., Fallon, J.F., and Beachy, P.A. (2000). Hedgehog-Regulated Processing of Gli3 Produces an Anterior/Posterior Repressor Gradient in the Developing Vertebrate Limb. Cell 100, 423-434.

Wang, Y., Li, X., and Hu, H. (2014). H3K4me2 reliably defines transcription factor binding regions in different cells. Genomics 103, 222-228.

Welscher, P. te, Zuniga, A., Kuijper, S., Drenth, T., Goedemans, H.J., Meijlink, F., and Zeller, R. (2002). Progression of Vertebrate Limb Development Through SHH-Mediated Counteraction of GLI3. Science 298, 827-830.

te Welscher, P., Fernandez-Teran, M., Ros, M.A., and Zeller, R. (2002). Mutual genetic antagonism involving GLI3 and dHAND prepatterns the vertebrate limb bud mesenchyme prior to SHH signaling. Genes Dev 16, 421-426. 
Wen, X., Lai, C.K., Evangelista, M., Hongo, J.-A., de Sauvage, F.J., and Scales, S.J. (2010). Kinetics of Hedgehog-Dependent Full-Length Gli3 Accumulation in Primary Cilia and Subsequent Degradation. Mol Cell Biol 30, 1910-1922.

Wyngaarden, L.A., Delgado-Olguin, P., Su, I. -hsin, Bruneau, B.G., and Hopyan, S. (2011). Ezh2 regulates anteroposterior axis specification and proximodistal axis elongation in the developing limb. Development 138, 3759-3767.

Yelon, D., Ticho, B., Halpern, M.E., Ruvinsky, I., Ho, R.K., Silver, L.M., and Stainier, D.Y. (2000). The bHLH transcription factor hand2 plays parallel roles in zebrafish heart and pectoral fin development. Development 127, 2573-2582.

Zhang, T., Cooper, S., and Brockdorff, N. (2015). The interplay of histone modifications - writers that read. EMBO Rep 16, 1467-1481.

Zhang, Y., Liu, T., Meyer, C.A., Eeckhoute, J., Johnson, D.S., Bernstein, B.E., Nusbaum, C., Myers, R.M., Brown, M., Li, W., et al. (2008). Model-based Analysis of ChIP-Seq (MACS). Genome Biology 9, R137.

Zhang, Z., Feng, J., Pan, C., Lv, X., Wu, W., Zhou, Z., Liu, F., Zhang, L., and Zhao, Y. (2013). Atrophin-Rpd3 complex represses Hedgehog signaling by acting as a corepressor of CiR. J Cell Biol 203, 575-583.

Zhu, J., Nakamura, E., Nguyen, M.-T., Bao, X., Akiyama, H., and Mackem, S. (2008). Uncoupling Sonic hedgehog control of pattern and expansion of the developing limb bud. Dev Cell 14, 624-632.

Zhulyn, O., Li, D., Deimling, S., Vakili, N.A., Mo, R., Puviindran, V., Chen, M.-H., Chuang, P.-T., Hopyan, S., and Hui, C. (2014). A switch from low to high Shh activity regulates establishment of limb progenitors and signaling centers. Dev Cell 29, 241249.

Zuniga, A., and Zeller, R. (2020). Dynamic and self-regulatory interactions among gene regulatory networks control vertebrate limb bud morphogenesis. Curr Top Dev Biol 139, 61-88. 


\section{Figure Legends}

Figure 1. GLI3 binds to poised, accessible chromatin prior to HH signaling. A. schematic for $\mathrm{HH}$ induction timeline during limb development. B. Quantification of 21-25S embryos with expression of Gli1 and Shh assayed by in situ hybridization. C. Representative Western blot $(n=3)$ of endogenous GLI3 ${ }^{F L A G}$ protein in the limb bud pre-(21-23S) and post-HH signaling (3235S). D. Venn diagram of all pre- and post-HH identified GLI3 CUT\&RUN called peaks. E. Percentage of E10.5 GLI3-bound regions also bound at E9.25, before $\mathrm{HH}$ signaling, for all E10.5 identified GBRs and E10.5 HH-responsive GBRs. F. Venn diagram of HH-responsive GBRs enriched for poised enhancer marks and bound by GLI3 at E9.25. Poised enhancer modifications identified by H3K4me1 CUT\&Tag ( $n=3)$, H3K4me2 ChIP-seq ( $n=2)$, ATAC-seq $(n=2)$. G. Heatmap of GLI3 enrichment pre- and post-HH signaling and enrichment of poised enhancer marks pre-HH signaling. Many regions lacking GLI3 also lack enrichment of poised marks. $\mathrm{H}$. Example of a HH-responsive GBR (orange shading) that is poised, accessible, and bound by GLI3 prior to HH signaling at a limb-specific Ptch1 enhancer (Lopez-Rios et al., 2014). I. Example of a validated $\mathrm{HH}$-responsive GBR GRE1, that regulates the GLI3 target Gremlin (Li et al., 2014), that is inaccessible, lacks H3K4me1 and GLI3 binding at E9.25. Scale bars $=1 \mathrm{~kb}$. See also Figure S1, Supplemental File 1,2.

\section{Figure 2. GLI3 binding regions have reduced enrichment of H3K27ac and are enriched} with HDACs in pre-HH limb buds. A. H3K27ac ChIP-seq tracks showing a E9.25 (21-23S) GLI3-bound HH-responsive GBR with reduced acetylation in E9.25 WT limb buds compared to E10.5 WT limbs (note that the levels of enrichment are comparable to E10.5 Shh ${ }^{-/}$limbs). B. Scatter plot of H3K27ac enrichment at HH-responsive GBRs in pre- and post-HH WT limbs. HHresponsive GBRs with significant reductions (FDR<0.05) in H3K27ac at E9.25 compared to 
E10.5, are denoted in dark blue, while the 3 GBRs with significantly higher H3K27ac in E9.25 limbs are in orange $(F D R<0.05 n=2)$. C. Heatmap indicating relative $\mathrm{H} 3 \mathrm{~K} 27$ ac enrichment at $\mathrm{HH}$ responsive GBRs in pre- and post-HH limbs (n=2). D. GLI3, HDAC1 and HDAC2 CUT\&RUN tracks at E9.25 GLI-bound regions, pre- and post-HH. E. HDAC localization at E10.5 bound GBRs. Prior to HH signaling, most regions are enriched for both HDAC1 and HDAC2, while after, most GBRs are enriched for only HDAC1 (E9.25 HDAC1/2 n=2; E10.5 HDAC1/2 n=3). F. Initially most HDAC1-bound regions are also enriched for HDAC2, while after $\mathrm{HH}$-signaling, fewer HDAC1-bound regions are also enriched for HDAC2. G. Prior to HH signaling, GLI3bound regions are enriched for poised enhancer marks with lower levels of H3K27ac and HDAC enrichment. These are comparable to post-HH GLI3 enhancers where GLI3 actively represses targets. Orange shading in tracks indicates HH-responsive GBRs defined in Figure S1C. Scale bars $=1 \mathrm{~kb}$. See also Figure S2, Supplemental File 1,2.

Figure 3. Loss of Gli3 does not result in premature de-repression of enhancers. A.

Schematic for testing whether loss of Gli3 prematurely increases H3K27ac levels through GLI3 de-repression. B. Heatmap of H3K27ac enrichment at HH-responsive GBRs in pre-HH (E9.25, 21-23S) and post-HH (E10.5, 33-34S) limb buds, with loss of Gli3. At E10.5, Shh/ limbs have reduced H3K27ac due to GLI3 repression. Loss of Gli3 in Shh ${ }^{-/}$; Gli3 ${ }^{-/}$results in de-repression of target enhancers and increased acetylation. This change is not observed prior to $\mathrm{HH}$ signaling with loss of Gli3 compared to WT limb buds. C. Scatterplot of H3K27ac enrichment in pre-HH WT vs. Gli3 ${ }^{-/}$limbs shows no increase in acetylation with loss of Gli3. D. H3K27ac ChIP-seq tracks at a pre-HH GLI3-bound HH-responsive GBR with low H3K27ac in E9.25 WT limb buds that does not increase acetylation levels in E9.25 Gli3- E. Examples of regions, bound by GLI3 at E9.25, that do not increase H3K27ac levels with loss of Gli3 in pre-HH limb buds as they do in $\mathrm{Shh}^{-/} ;$Gli3 ${ }^{-/}$post-HH limbs. F. Schematic depicting loss of Gli3 in pre-HH limb buds does not 
lead to de-repression of target enhancers as it does after $\mathrm{HH}$ induction at E10.5. Orange shading on tracks indicates HH-responsive GBRs defined in Figure S1C. Scale bars $=1 \mathrm{~kb}$. See also Supplemental File 1,2.

\section{Figure 4. Most genes are not de-repressed in Gli3 mutants prior to HH induction. A.}

Schematic for testing whether loss of Gli3 can prematurely activate target genes through GLI3

de-repression. B,C. Volcano plot of differentially expressed genes (DEGs) detected in RNA-seq between WT and Gli3- limbs E9.25 (21-23S) and E10.5 (32-35S) anterior limb buds. D.

Fluorescent in situ hybridization showing maximum intensity projections for Hand2, Foxf1 and Ptch1 in E9.25 WT and Gli3 $\%$ limb buds indicating the absence of detectable Foxf1 and Ptch1 in pre-HH limb buds. Dashed white lines outline the limb bud region. E. In situ hybridization for the GLI3 target Hand2, in WT and Gli3 ${ }^{-/}$limbs pre- $(21,23 S)$ and immediately post-HH induction (24-27S; $n=3$ ). Note that Hand2 is expressed almost uniformly across the limb bud in $21 \mathrm{~S}$ embryos in both WT and Gli3 ${ }^{--}$limbs. At 23S, slight reduction in anterior expression of Hand2 is observed in both WT and Gli3 ${ }^{-\%}$ limbs (white arrowhead). The red brackets denote anterior limb. F. Schematic identifying H3K27me3 enriched regions in E9.25, E10.5 anterior (GLI3-repressed) and E10.5 posterior ( $\mathrm{HH}$ signaling, loss of GLI-R) limb buds using CUT\&Tag. G-J Orange shading in tracks indicates $\mathrm{HH}$-responsive GBRs defined in Figure S1C. G. Example of a HHresponsive GBR at a $\mathrm{HH}$ target gene promoter with $\mathrm{H} 3 \mathrm{~K} 27$ me3 enrichment specifically at E9.25 but not E10.5. H. Representative distal limb-specific HH-responsive GBR downstream of Ptch1 with no H3K27me3 enrichment in E9.25 or E10.5 limb buds. I. Examples of HH target genes containing polycomb repression that could potentially be incompetent for GLI3 repression. J. Examples of genes that are bound by GLI3 at E9.25, contain H3K27ac enrichment and lack H3K27me3 enrichment, suggesting they are competent for GLI3 repression but are not derepressed in the absence of Gli3. Scale bars for tracks indicate $1 \mathrm{~kb}$. See also Figure S3,S4, Supplemental File 2,3. 
Figure 5. GLI3-dependent chromatin compaction occurs after HH induction. A. ATAC-seq

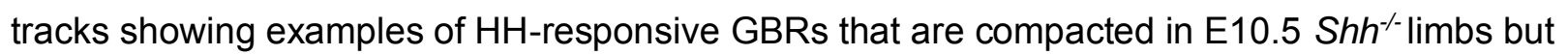

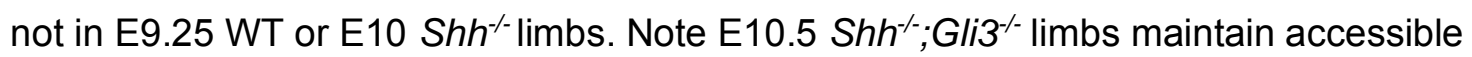
chromatin at these regions. B. Violin plots of chromatin accessibility in WT and Shh ${ }^{-/}$limbs. Globally the chromatin at $\mathrm{HH}$-responsive GBRs is more accessible in E10 Shh-/ limb buds compared to E10 WT limbs and E10.5 Shh ${ }^{-/}$limbs (n=2-3 at each stage). Wilcoxon signed rank tests were performed to compare each pair of groups, multiple hypothesis testing adjusted using BH method. E9.25<E10 WT, FDR<2.69E-15; E9.25<E10 Shh ${ }^{-/}$FDR<3.44E23; E9.25<E10.5 FDR<5.82E-15 E10 WT<E10 Shh ${ }^{-/,}$FDR<2.55E-4; E10 Shh ${ }^{-/}>$E10.5 Shh $h^{-/}$, FDR<1.21-18; E10.5 WT>E10.5 Shh ${ }^{-/}$, FDR<9.81E-12). C. Average ATAC profiles of HHresponsive GBRs in E10 and E10.5 Shh ${ }^{-/}$limb buds. D. Violin plots depicting log2 fold changes in chromatin accessibility (WT versus Shh $\left.{ }^{-/}\right)$at E10 and E10.5 E ( $p<0.0001$ Wilcoxon signed rank test). E,F. Scatter plots of ATAC-seq signal in WT vs. Shh $h^{-/} \operatorname{limbs}$ at E10 (E) and E10.5 (F). GBRs and their associated genes annotated in red signify a significant reduction of chromatin accessibility in $S h h^{-/}$limbs compared to WT (FDR<0.05). GBRs in blue indicate visibly reduced accessibility in E10 Sh/ ${ }^{-/}$limb buds that are not significantly changed until E10.5 G. ATAC-seq tracks of GBRs near Ptch1 that are reduced, but not significant, in E10 Shh ${ }^{-/}$limbs compared to E10 WT limb buds. H. GBRs that are already have significantly reduced accessibility in E10 Shh/ limbs compared to E10 WT. J. Schematic showing the temporal onset of chromatin compaction at GBRs in relation to the initiation of $\mathrm{HH}$ signaling. K. Quantification of GLI3 present along ciliary axonemes out of total number of cilia (marked by ARL13b) that colocalize with GLI3 in E9.25 (21-23S) (n=3), E9.75 (26-28S) center limb buds ( $n=6)$ and E10.5 (35S) anterior and posterior limbs $(n=3)$. Error bars indicate SEM. L. Representative images of GLI3 ${ }^{\text {FLAG }}$ ciliary distribution in E9.25 and E9.75 limb buds acquired on a Zeiss LSM 710 
Confocal visualized using maximal intensity projections. N. Schematic depicting the temporal shift in GLI3 ciliary localization. Orange shading in all tracks indicates HH-responsive GBRs defined in Figure S1C. Scale bars for ATAC-seq tracks $=1 \mathrm{~kb}$. Scale bars for panel $\mathrm{L}=0.5 \mu \mathrm{m}$. See also Figure S5, Supplemental File 4.

Figure 6. Model for established GLI3-mediated repression. A. Comparison of expression levels of predicted GLI target genes (Supplemental File 3), with pre-HH H3K27ac enrichment at promoters (60/74 genes) in pre-(21-23S) and post-HH (32-35S) limb buds. Grey dashed lines are indicative of minimal expression thresholds, $\log 2(T P M+1)<0.5$. Note that most GLI target genes are already transcribed in pre-HH limb buds. B. Model for lack of GLI3 repression prior to $\mathrm{HH}$ signaling. In pre- $\mathrm{HH}$ limb buds, many target genes are already activated by $\mathrm{HH}$-independent factors. While GLI3 binds to many (but not all) targets, it appears inert as it is unable to regulate deposition of H3K27ac at enhancers or chromatin accessibility as it does in later limb development. After HH induction when ‘active’ GLI3 repression has been established, GLI3 prevents addition of $\mathrm{H} 3 \mathrm{~K} 27 \mathrm{ac}$ at enhancers to spatially restrict expression of its target genes and pattern the limb. C. Schematic of GLI3 spatial regulation of target genes. In pre-HH limb buds, GLI3 does not repress targets such as Hand2, which is expressed in an expanded anterior domain throughout the limb bud. In contrast, GLI3 restricts expression of targets to the posterior limb bud where $\mathrm{HH}$ signaling is active in post-HH limb buds. D. Possible models for initiating GLI3 repressor activity. Top: $\mathrm{HH}$-independent co-repressor(s), which may not be abundant in early limb development collaborate with GLI3 to assemble a complete repression complex. Middle: Addition of post-translational modifications (PTMs), potentially added via ciliary trafficking and processing, promote assembly of, or stabilize, a GLI repression complex. Bottom: A spike in ciliary GLI3 processing, as reflected by increased axonemal colocalization, increases the amount of viable GLI3 repressor, potentially through addition of post-translation modifications as suggested above. 


\section{Supplemental Tables}

Supplemental Table 1. Predicted direct GLI3 target genes (tab 1). Predicted direct target genes enriched with H3K27me3 and H3K27ac in pre-HH limb buds (tab 2).

\section{Supplemental Data Files}

Supplemental File 1. Called peaks for CUT\&RUN and CUT\&Tag datasets. Includes: E9.25 and anterior E10.5 GLI3 CUT\&RUN, E9.25 H3K4me1 CUT\&Tag, E9.25 H3K27me3 CUT\&Tag, E10.5 anterior and posterior H3K27me3 CUT\&Tag, E9.25 HDAC1, E9.25 HDAC2, E10.5 HDAC1, E10.5 HDAC2.

Supplemental File 2. Differential Chip-seq analyses. Differential analysis of H3K4me2 ChIP-seq enrichment in E9.25 (21-23S) and E10.5 (32-35S) WT limb buds (tab 1). Differential analysis of H3K27ac ChIP-seq enrichment in E9.25 (21-23S) vs. E10.5 (3235S) WT limb buds (tab 2) and E9.25 (21-23S) WT vs. Gli3\% limb buds (tab 3).

Supplemental File 3. Differentially expressed genes in RNA-seq datasets. Differentially expressed genes in WT vs. Gli3/- E9.25 limb buds (tab 1), WT vs. Gli3/- E10.5 anterior limb buds (tab 2) and E9.25 vs. E10.5 anterior WT limb buds (tab3). Intron retention rates in E9.25 Gli3\% limb buds and E10.5 Gli3\% limb buds (tab 3). 'IRtype' denotes if a gene has a significantly higher IR rate at E9.25, E10.5 or neither (labeled 'other').

Supplemental File 4. Differential ATAC-seq comparing E9.25 WT whole limb buds, E10 WT (28-30S), E10 Shh ${ }^{-/}$(28-30S), E10.5 WT (35S) and E10.5 Sh/ ${ }^{-/}$posterior limb buds. 
A.

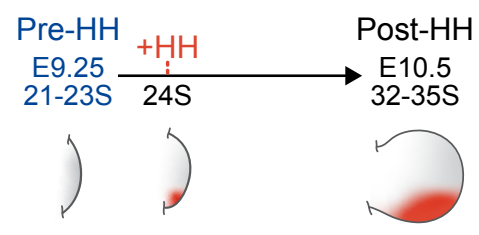

B.

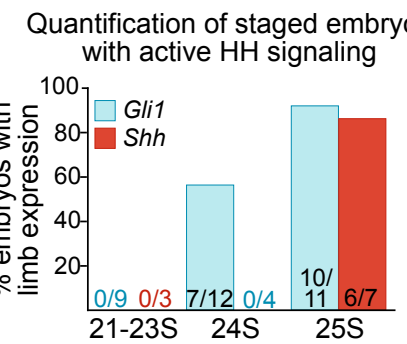

F.

E9.25 GLI-bound regions enriched for poised enhancer modifications

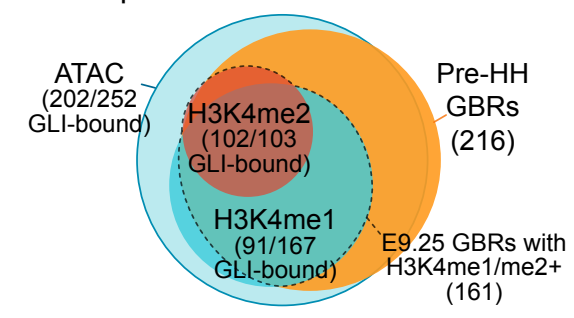

G.

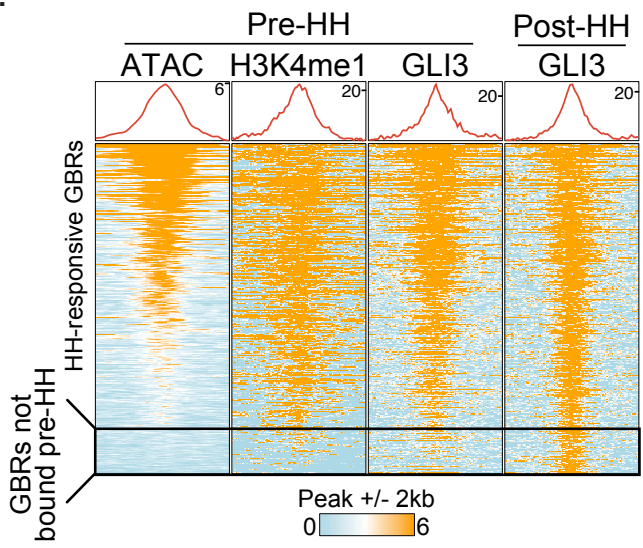

C.

$$
\begin{aligned}
& \text { Pre-HH Post-HH } \\
& \text { E9.25 E10.5 }
\end{aligned}
$$

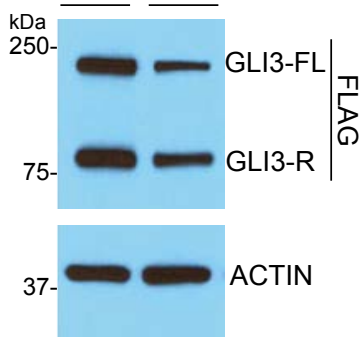

$\mathrm{H}$.

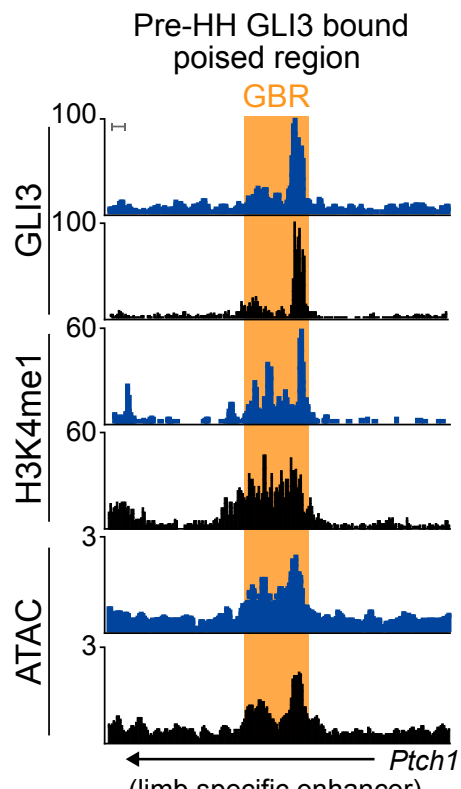

(limb specific enhancer)

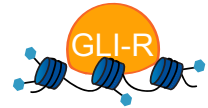

GLI-bound Enhancer H3K4me1/2 accessible chromatin
E.

Post-HH GBRs bound by GLI3 Pre-HH
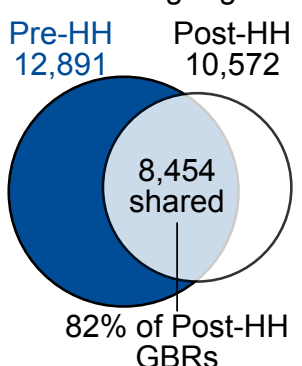

I.

Pre-HH GLI3 unbound region

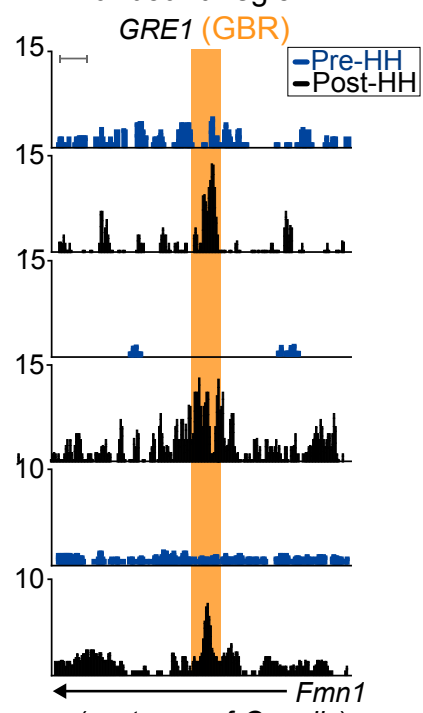

(upstream of Gremlin)

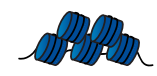

Unbound GLI Enhancer No H3K4me1/2 inaccessible chromatin

Figure 1. GLI3 binds to poised, accessible chromatin prior to $\mathrm{HH}$ signaling. 
A.

HH-responsive GBR

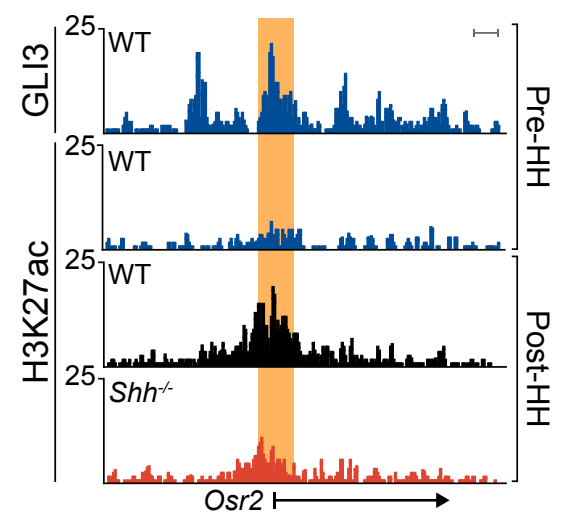

D.

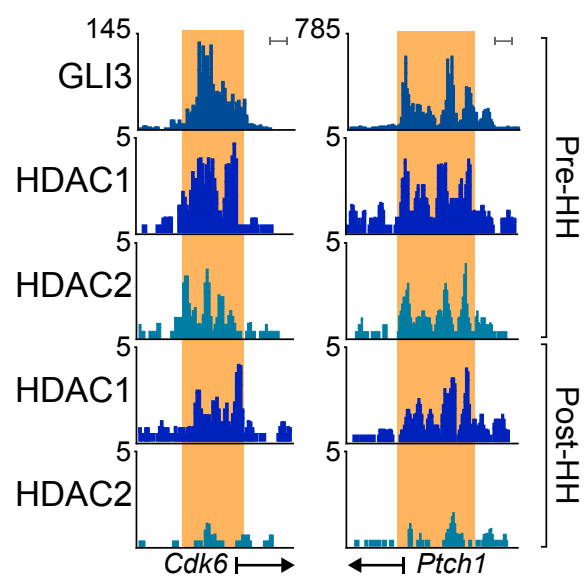

B.

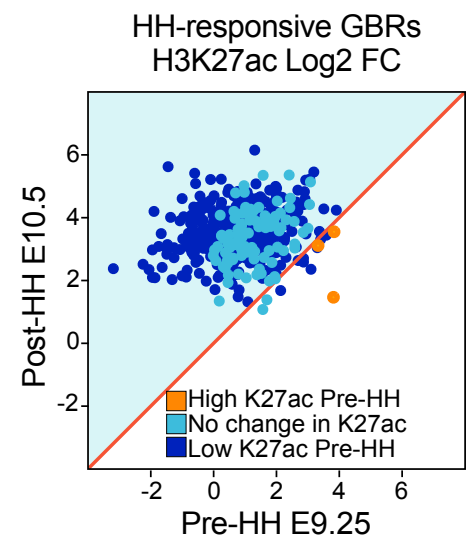

E.



F. HDAC co-localization at GBRs

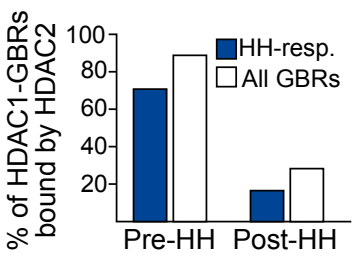

C.

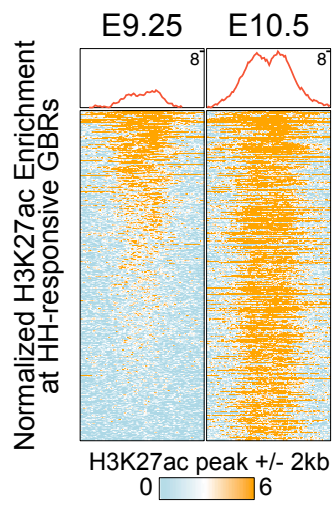

G.

Pre-HH (GLI Repression?)

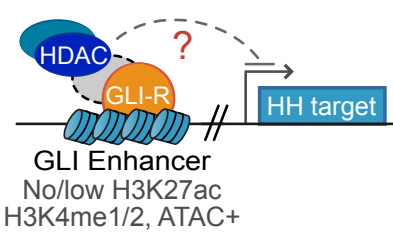

Post HH (GLI Repression)

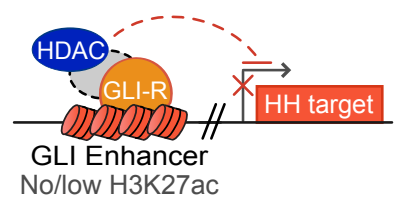

Figure 2. GBRs have reduced H3K27ac and are enriched with HDACs in the early limb. 
A.

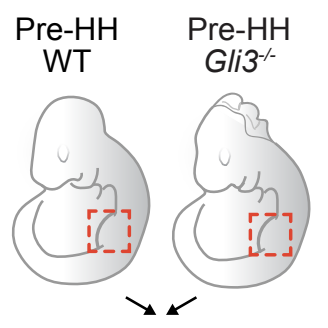

H3K27ac ChIP-seq

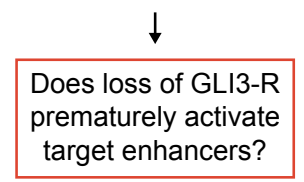

B.

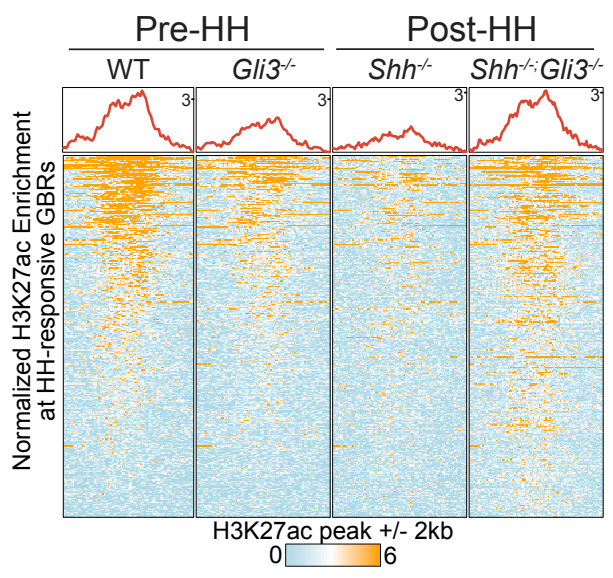

C.

Pre-HH H3K27ac Log 2 FC

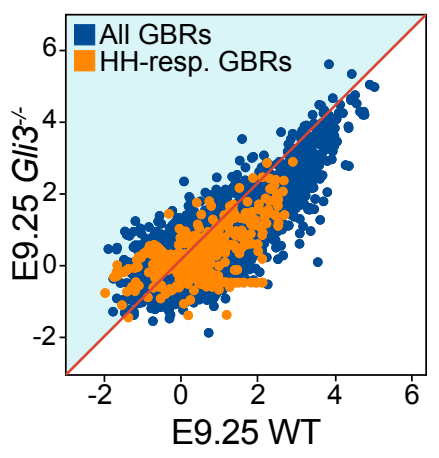

F.
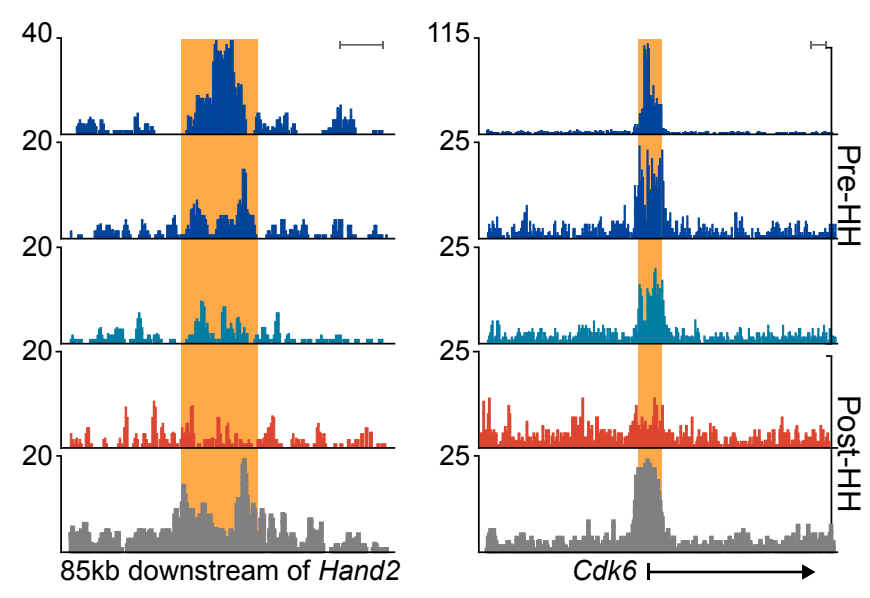

D.

Pre-HH bound GBRs

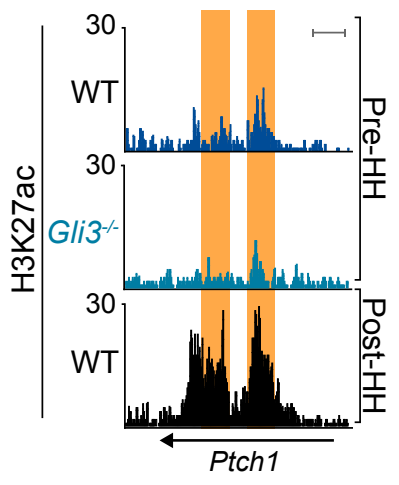

E.

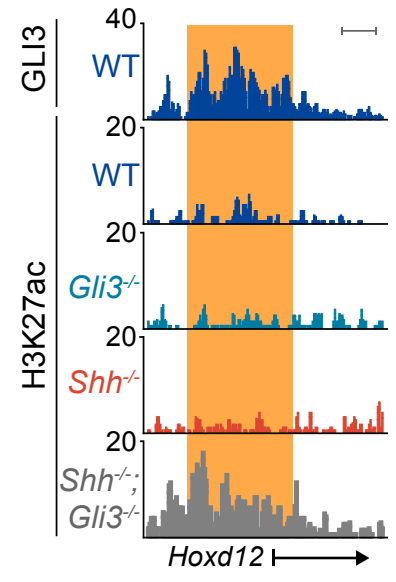

Pre-HH

Loss of GLI3 does not de-repress enhancers

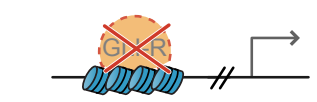

GLI Enhancer No/low H3K27ac

\section{Post-HH}

Loss of GLI3 leads to de-repression

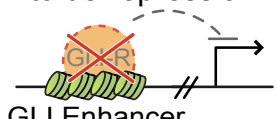

GLI Enhancer High H3K27ac

Figure 3. Loss of Gli3 does not result in premature de-repression of enhancers. 
A.

Pre-HH WT Pre-HH Gliß

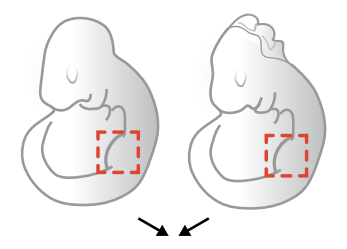

RNA-seq

$\downarrow$

Does loss of GLI3-R prematurely activate target genes?

E.

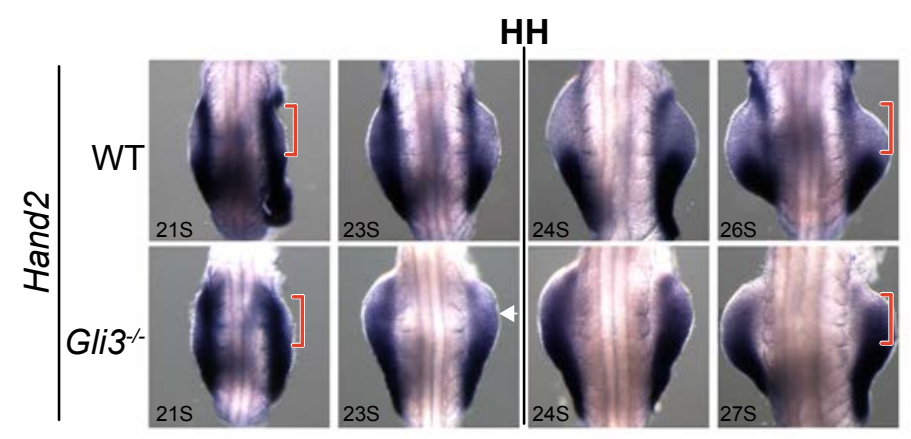

I.
B.

E9.25 Pre-HH Limbs

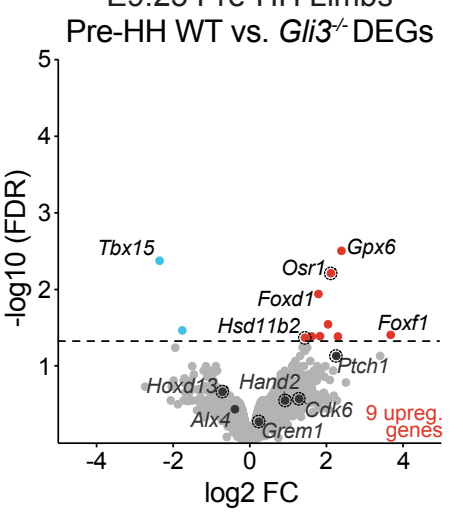

F.

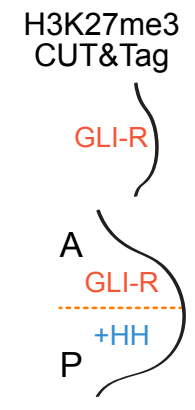

J.
D. Hand2/Ptch1 Hand2/Foxf1
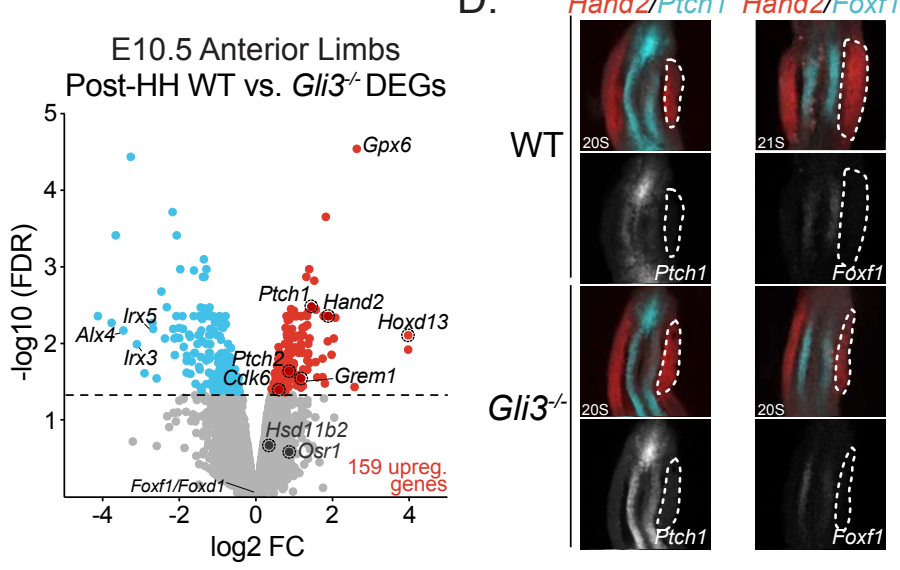

$\mathrm{H}$.

G.

Promoter GBR
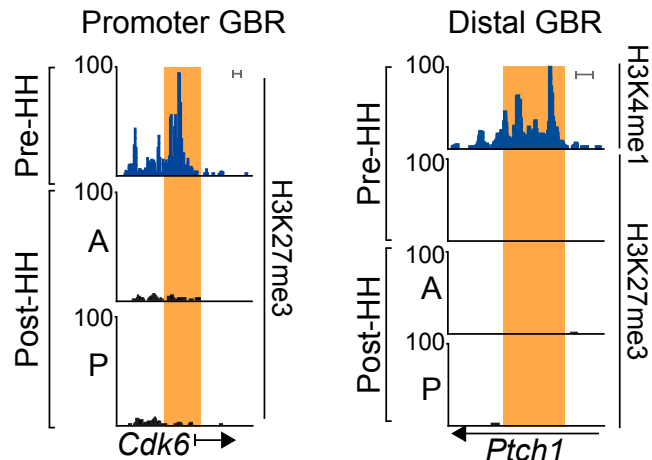

(37kb downstream)

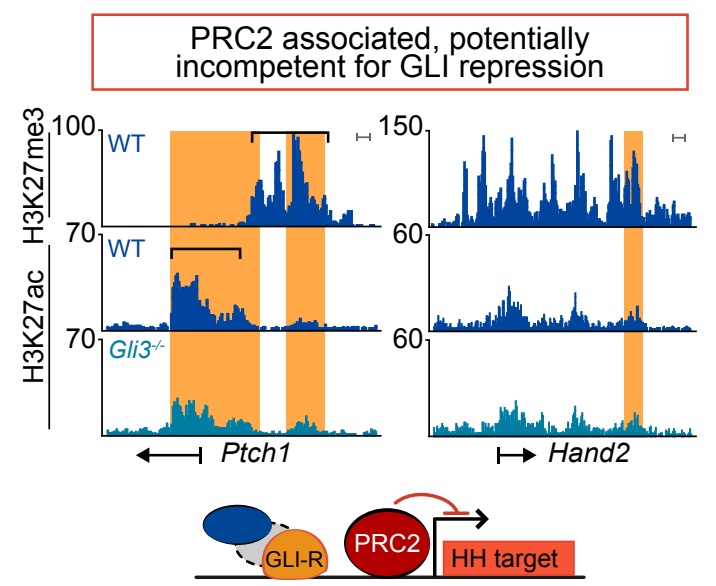

$18 \%$ of genes with $\mathrm{H} 3 \mathrm{~K} 27 \mathrm{me} 3$ only $47 \%$ of genes with H3K27me 3 \& H3K27ac

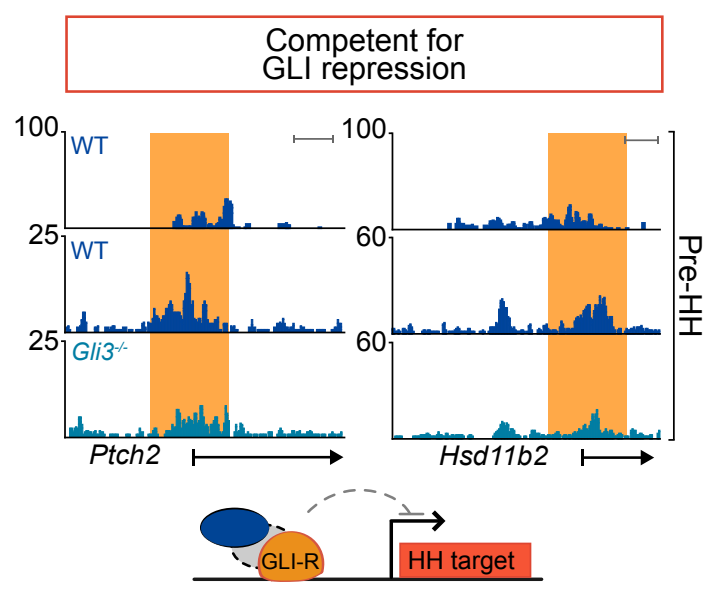

$34 \%$ of genes with $\mathrm{H} 3 \mathrm{~K} 27 \mathrm{ac}$ only

Figure 4. Most genes are not de-repressed in Gli3 mutants prior to HH induction. 
A.

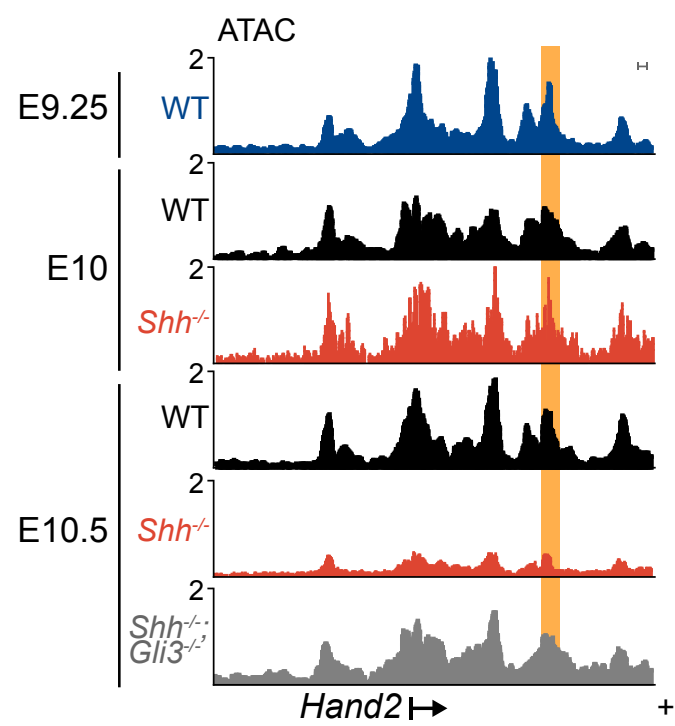

D.

WT vs Shh ${ }^{-1}$ Fold Enrichment at $\mathrm{HH}$-responsive GBRs

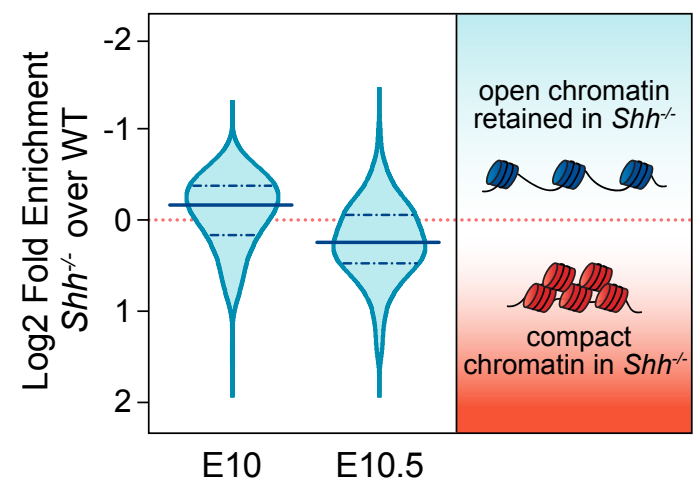

G.

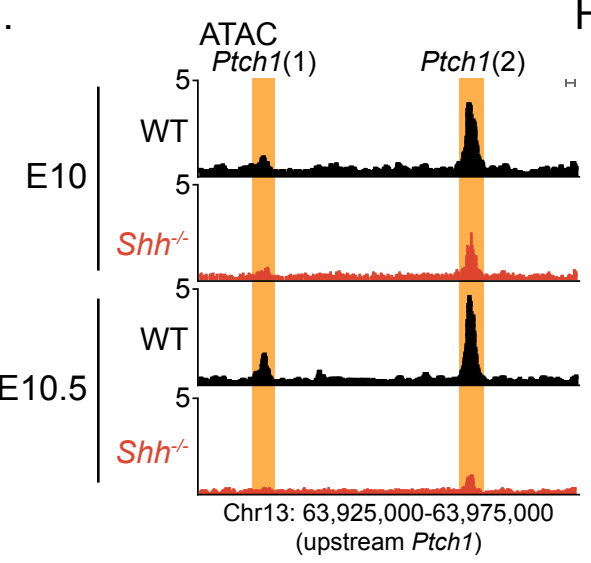

$\mathrm{H}$.

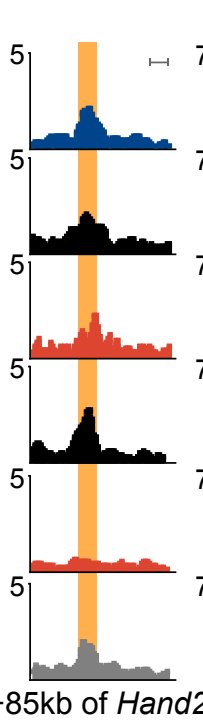

E.
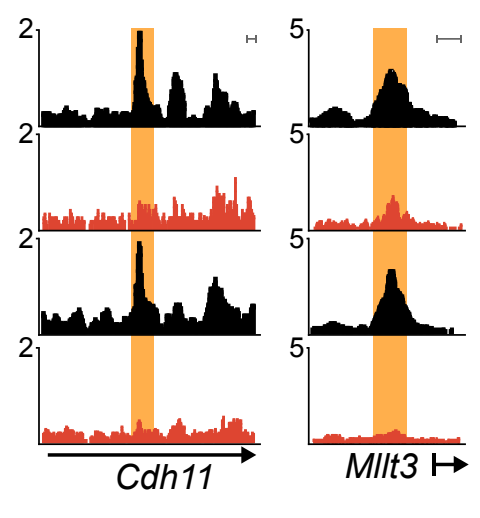

$\mathrm{K}$.

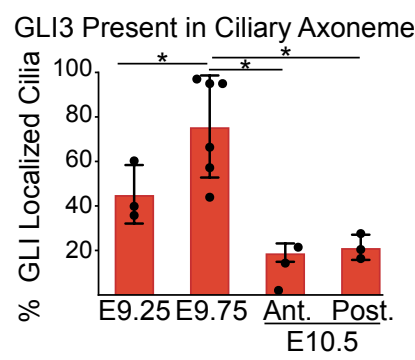

L.

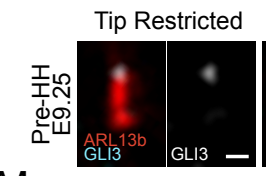

M.

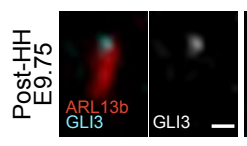

J.

N.
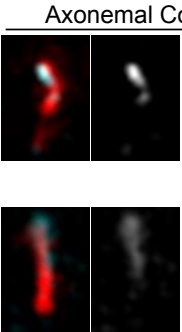

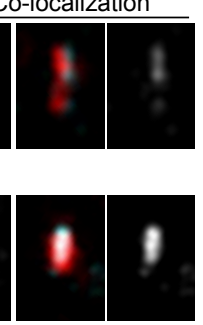

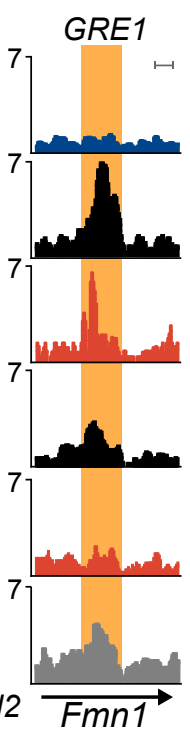

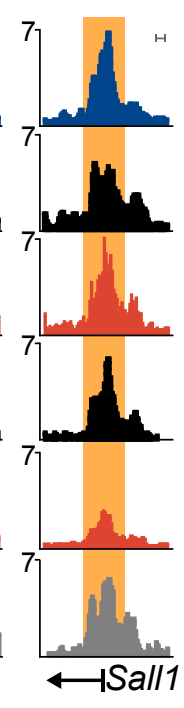

B.

HH-responsive GBRs

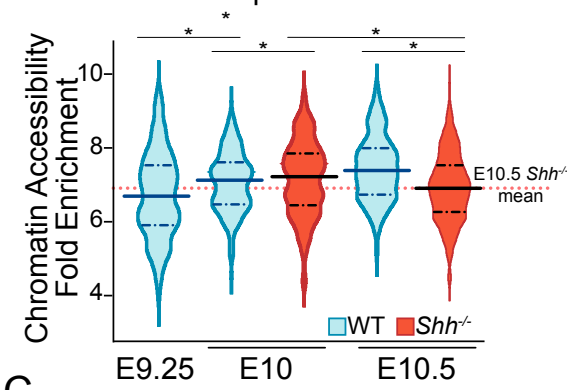

C.

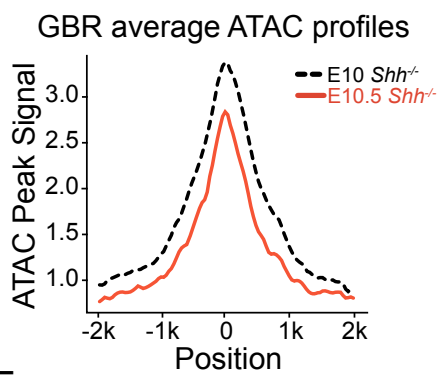

F.
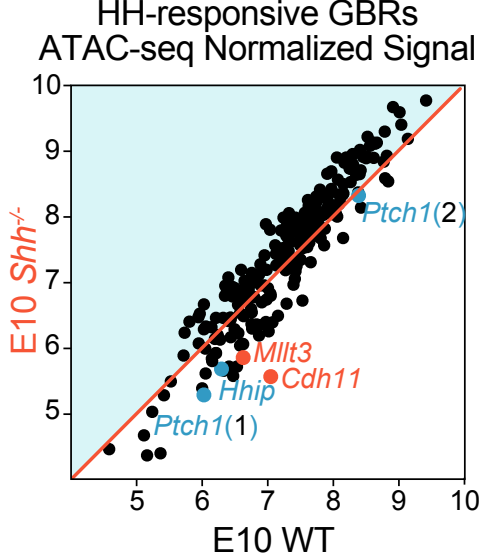

$\mathrm{HH}$-responsive GBRs

ATAC-seq Normalized Signal

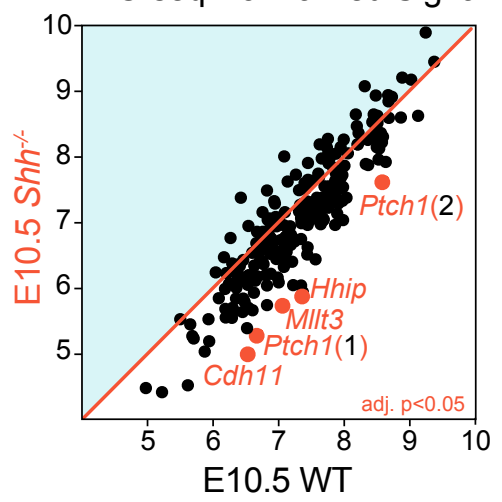

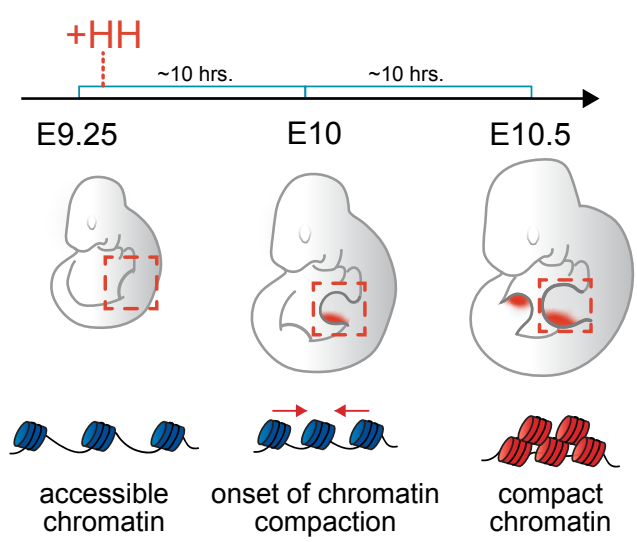

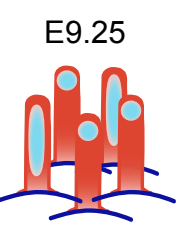

high GLI ciliary tip localization
E9.75

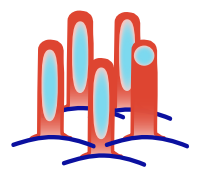

spike in GLI ciliary trafficking
E10.5

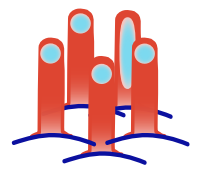

high GLI ciliary tip localization

Figure 5. GLI3-dependent chromatin compaction occurs after HH induction. 
bioRxiv preprint doi: https://doi.org/10.1101/2021.06.29.450392; this version posted June 29, 2021. The copyright holder for this preprint (which was not certified by peer review) is the author/funder. All rights reserved. No reuse allowed without permission.

A.

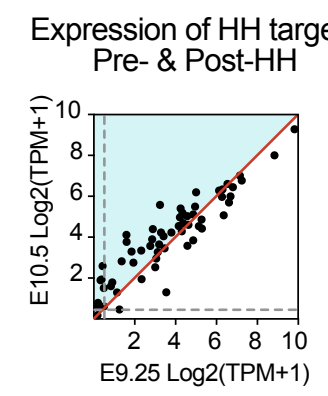

C.

Pre-HH, E9.25

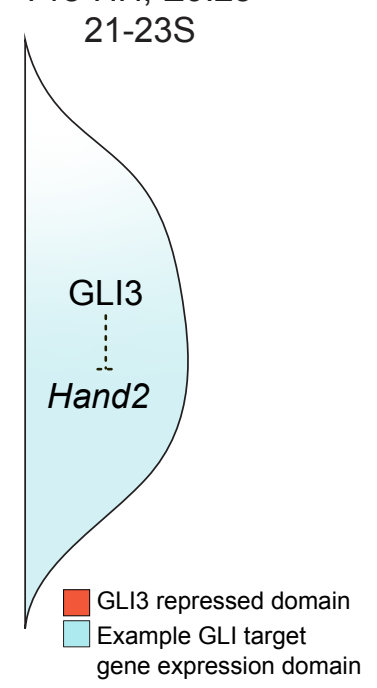

Pre-HH: Most genes activated prior to $\mathrm{HH}$ No ‘active' GLI repression

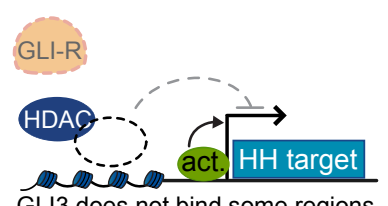

GLI3 does not bind some regions

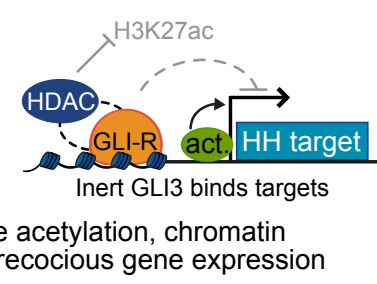

Post-HH: 'Acitve' GLI repression spatially restricts targets

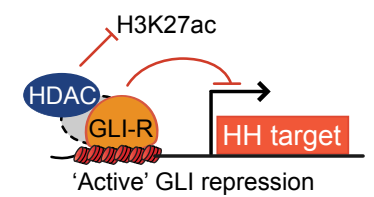

GLI3 prevents acetylation, enhancer activation \& gene expression
D. Models for establishing GLI repressor activity

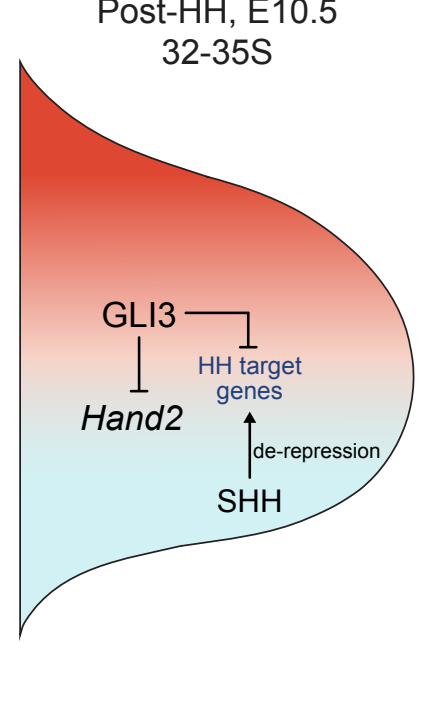

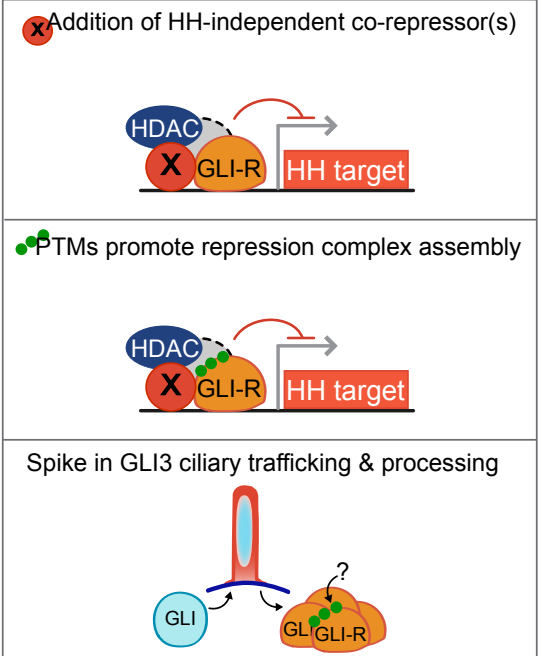

Figure 6. Model for established GLI3-mediated repression. 
A.

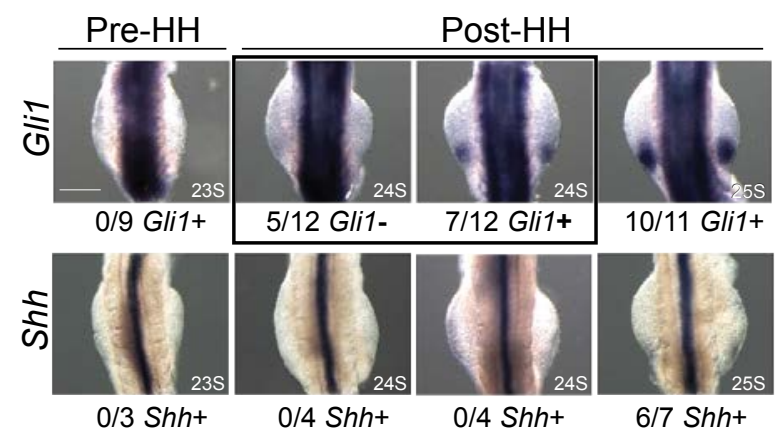

C.

HH-responsive H3K27ac at E10.5 GLI3 CUT\&RUN peaks

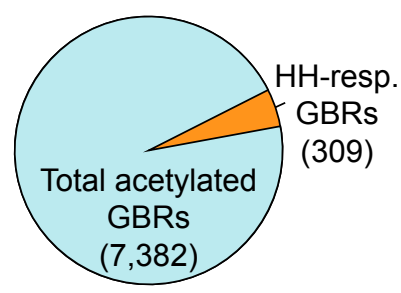

WT vs Shhr/ H3K27ac ChIP-seq peaks intersected with GLI3 Cut\&Run

G.

Poised enhancer modifications at $\mathrm{HH}$-resp. GBRs in pre-HH limbs

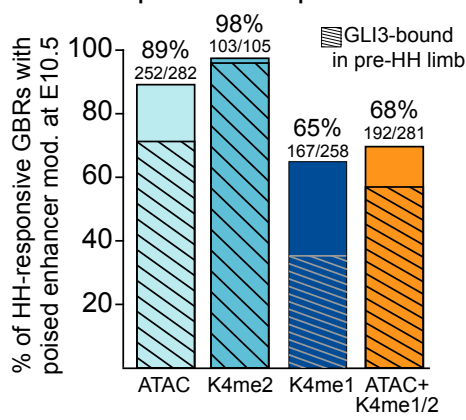

D.

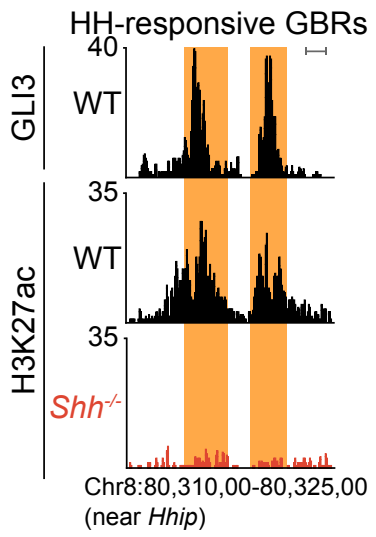

H.

Enhancer modifications at unbound $\mathrm{HH}$-resp. GBRs

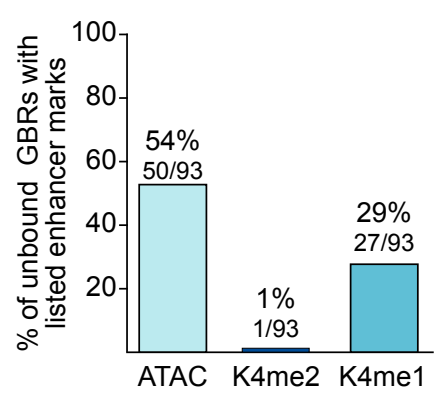

B.

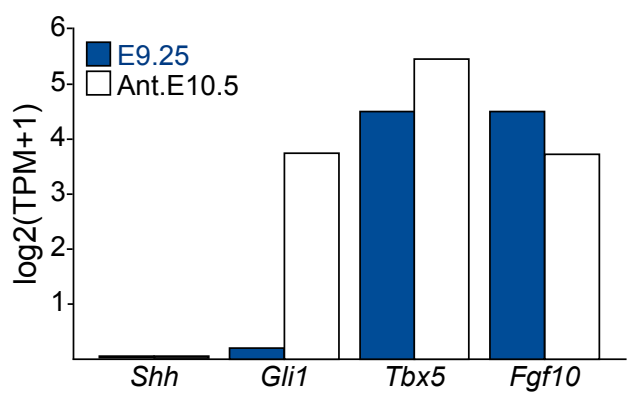

E.

F.

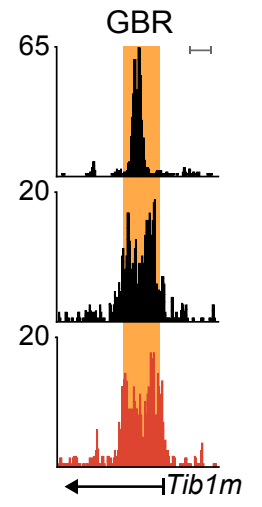

H3K4me2 at all Post-HH GBRs H3K4me2 log2 FC

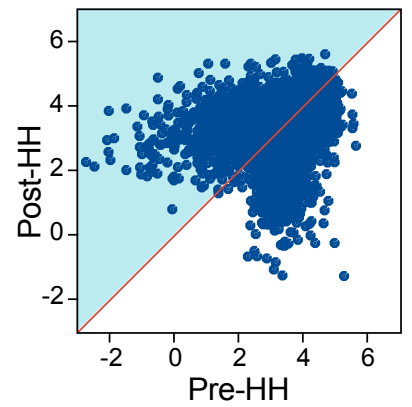

I.

Distribution of bound $\mathrm{HH}$-responsive GBRs from $\mathrm{HH}$ target gene TSSs

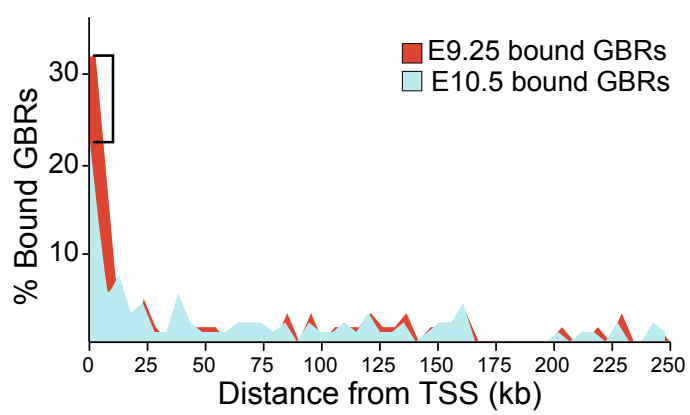

Figure S1. Properties of GLI3 binding at enhancers before and after the onset of HH signaling. A. Whole mount in situ hybridization for Gli1 and Shh in 23-25S embryos to define the onset of $\mathrm{HH}$ induction. While Shh is not detectable in the limb until 25S, the earliest detection of Gli1 is at 24S, where 5/15 embryos have Gli1 expression. B. Expression values for Shh, Gli1 and selected limb genes, in E9.25 and E10.5 anterior limb buds from RNA-seq data. C. Intersection of E10.5 GLI3 CUT\&RUN peaks with previously published WT vs Shh ${ }^{-/}$E10.5 H3K27ac ChIP-seq peaks (Lex et al., 2020). H3K27ac peaks reduced in Shh- limbs that overlap GLI3 binding regions were categorized as $\mathrm{HH}$-responsive GBRs. D, E. Examples of a HH-responsive GBR that loses acetylation in Shh ${ }^{-/}$limbs (D) and a GBR that maintains acetylation in the absence of $\mathrm{HH}$ signaling (E). Orange shading in tracks indicate the GBRs. F. Scatterplot showing H3K4me2 ChIP-seq fold enrichment at E10.5 GBRs in pre-HH (21-23S) and post-HH (32-35S) limb buds $(n=2$ biological replicates at each stage). No peaks were significantly changed between pre- and post-HH signaling. G. Percentage of $\mathrm{HH}-$ responsive GBRs enriched (called peaks) for the poised enhancer markers H3K4me1 (CUT\&Tag, $n=3$ ) and H3K4me2 $(n=2)$ and accessible chromatin (ATAC-seq peaks, $n=2$ ) prior to HH signaling. Percentages indicate the number of E9.25 GBRs enriched for the specified feature, out of the total number of $\mathrm{HH}$-responsive GBRs enriched for that enhancer modification at E10.5. H. Enhancer modifications present at HH-responsive GBRs that are not bound by GLI3 at E9.25. I. Distribution of E9.25 and E10.5 GBRs from the TSSs of putative $\mathrm{HH}$ target genes. Scale bars for tracks indicate $1 \mathrm{~kb}$. 
A.

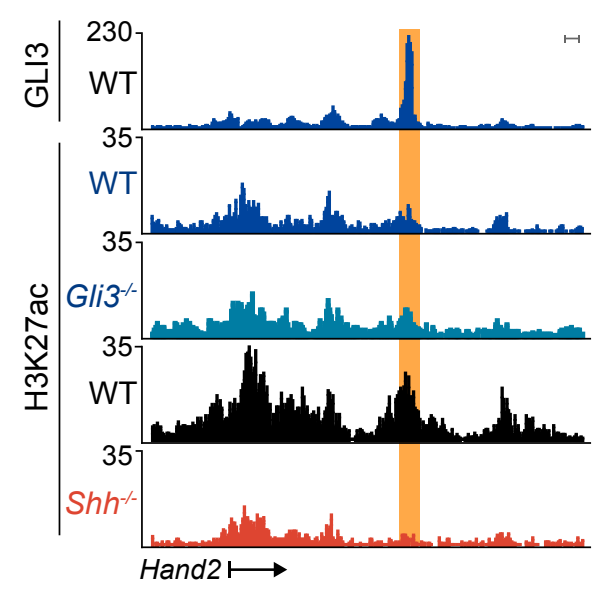

C.

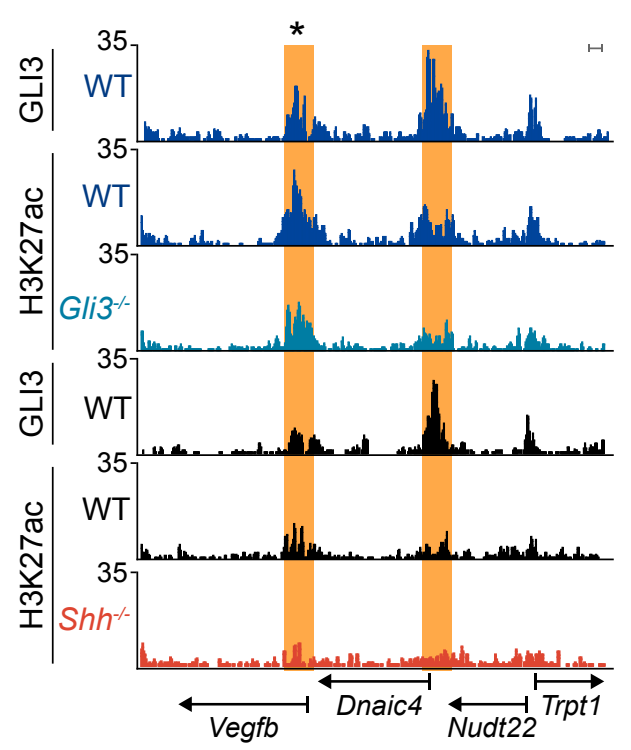

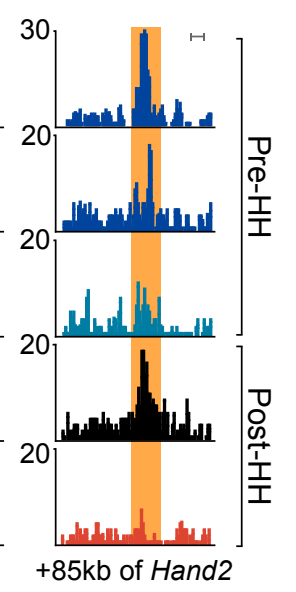

B.

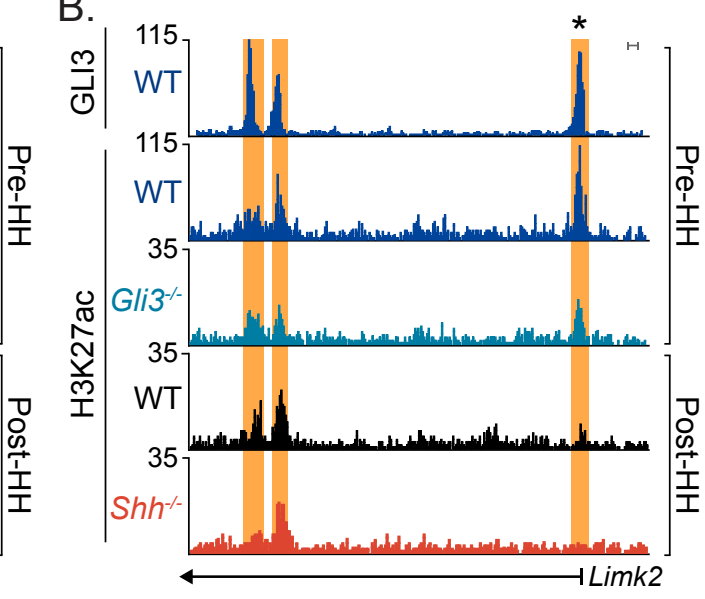

D.

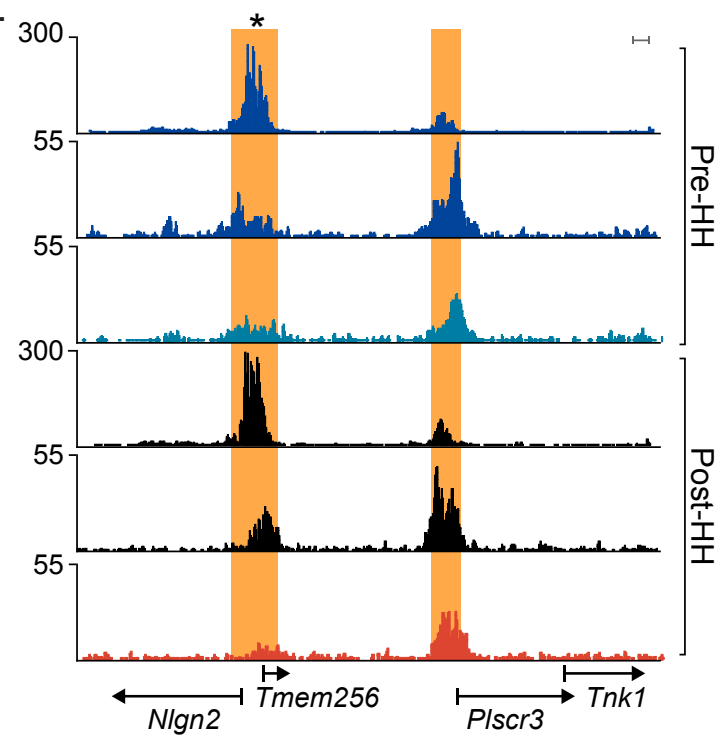

Figure S2. E9.25 acetylated GBRs that do not further increase H3K27ac levels with loss of Gli3. A. HH-responsive GBRs (orange shading) near the GLI3 target Hand2 that have H3K27ac enrichment at E9.25 do not have increased levels of H3K27ac in Gli3- limb buds, suggesting there is no de-repression of Hand2. B-D. $\mathrm{HH}$-responsive GBRs (orange shading) with significantly higher acetylation prior to $\mathrm{HH}$ induction (indicated by asterisk; FDR $<0.05$ ), do not have increased acetylation with the absence of Gli3. Scale bars for tracks indicate $1 \mathrm{~kb}$. 
A.

\begin{tabular}{|c|c|}
\hline Gene & Half-life(hrs) \\
\hline Gpx6 & 18.4 \\
\hline She & 2.87 \\
\hline Foxd1 & 3.16 \\
\hline Foxf1 & 2.58 \\
\hline Osr1 & 3.02 \\
\hline Mapre3 & 11.71 \\
\hline Cxcl13 & 24 \\
\hline Hsd11b2 & 24 \\
\hline Average & $\mathbf{1 1 . 2}$ \\
\hline Median & $\mathbf{7 . 4 3}$ \\
\hline
\end{tabular}

B.

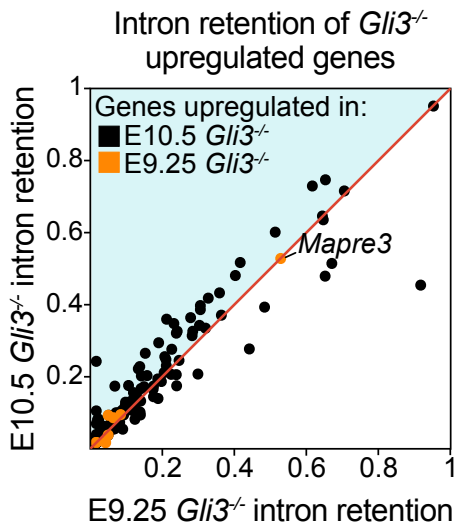

C.

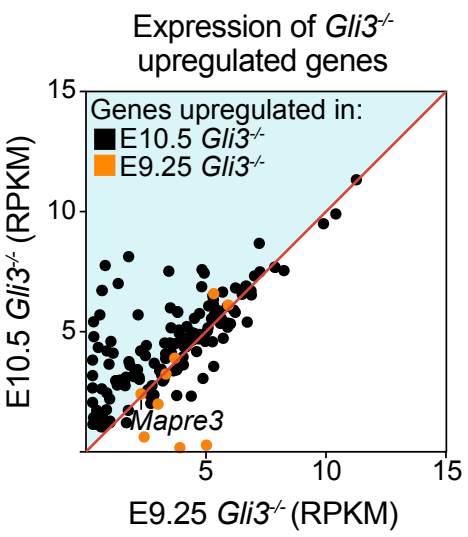

D.
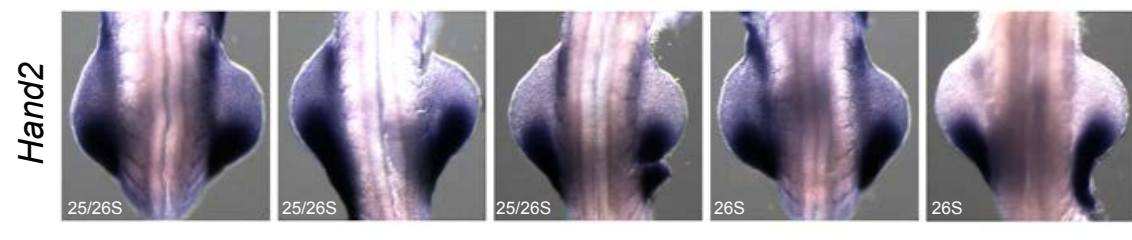

Figure S3. E9.25 Gli3/- upregulated genes are expressed at low levels and have Iow intron retention rates. A. Table of half-lives $\mathrm{E} 9.25 \mathrm{Gli}^{-1-}$ upregulated genes, previously determined in differentiating embryonic stem cells (Sharova et al., 2009). B. Intron retention levels in E9.25 and E10.5 Gli3/- upregulated genes. While E10.5 upregulated genes have intron retention rates that vary, E9.25 upregulated genes have intron retention rates near 0 , suggestive of mature transcripts. The exception to this is one gene, Mapre3, which has high intron retention in both E9.25 and E10.5 Gli3---, but is not upregulated at $\mathrm{E} 10.5$. The formula $(1-\mathrm{N}) / \mathrm{M}$ was used to calculate the intron retention rate for each gene, where $\mathrm{N}=$ the number of reads overlapping with any of the gene's exons and $M=$ the number of reads overlapping with the body of the gene. Limma (Ritchie et al., 2015) was used to identify differential intron retention rate between E9.25 and E10.5 $\mathrm{Gli3}^{-/}$limbs, FDR cutoff< 0.05. C. Expression of genes upregulated in E9.25 and E10.5 Gli3 ${ }^{-/}$limb buds. D. Expression of Hand2 in WT limb buds at $\sim 26 \mathrm{~S}$. Note that most embryos have some anterior expression of Hand2 and Hand2 is only completely posteriorly restricted in one embryo at this stage (far right). 
A.

$\mathrm{HH}$ target gene promoters enriched for H3K27me3

E9.25
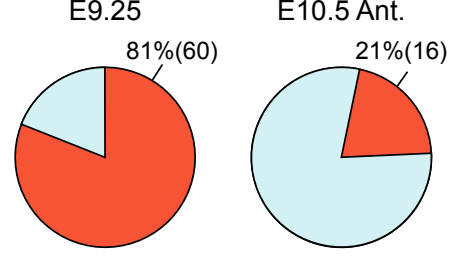

74 predicted direct $\mathrm{HH}$ target genes

B.

HH-resp. GBRs enriched for H3K27me3

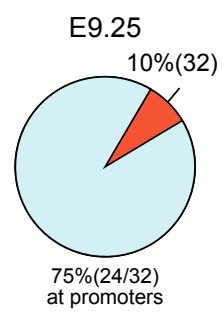

E10.5 Ant.

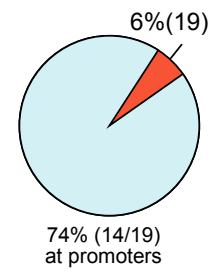

$309 \mathrm{HH}$-resp. GBRs

C.
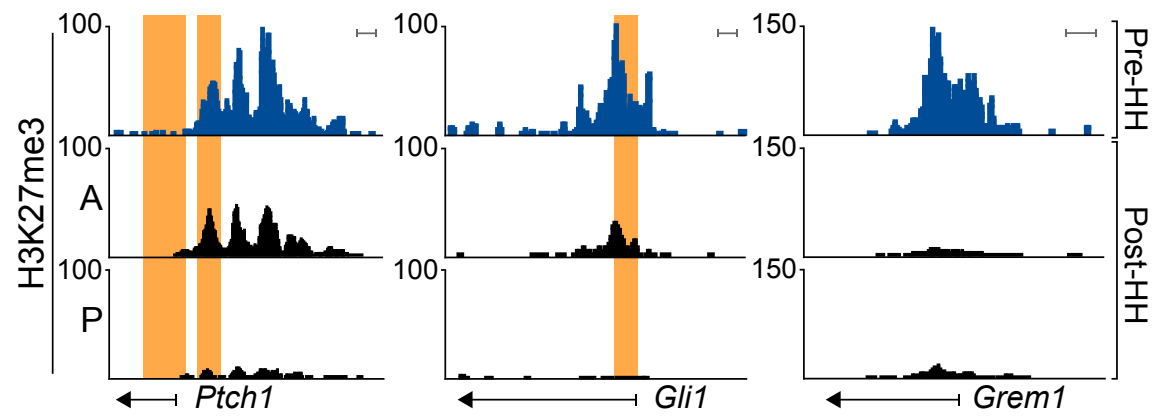

D.

Enrichment of H3K27ac at H3K27me3+ genes

E.

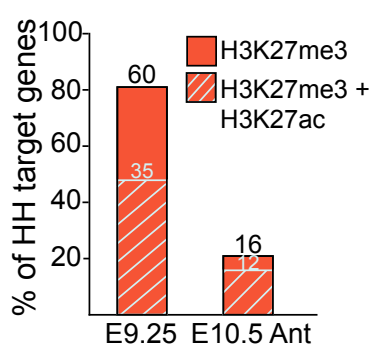

G.

H3K27me3+,
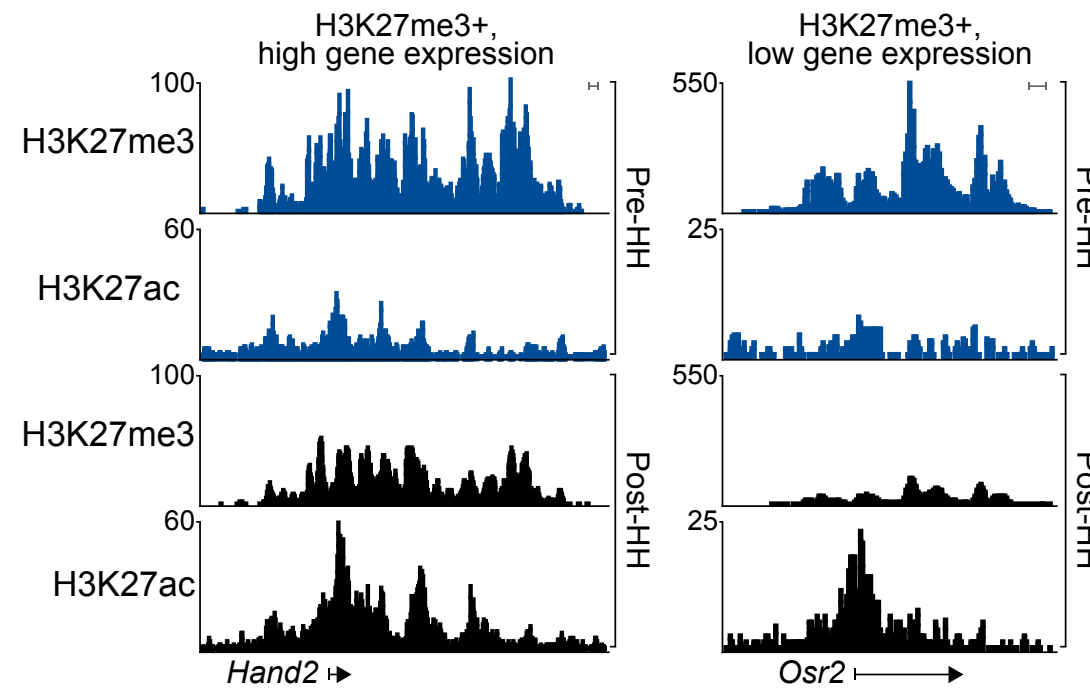

$\mathrm{H}$.

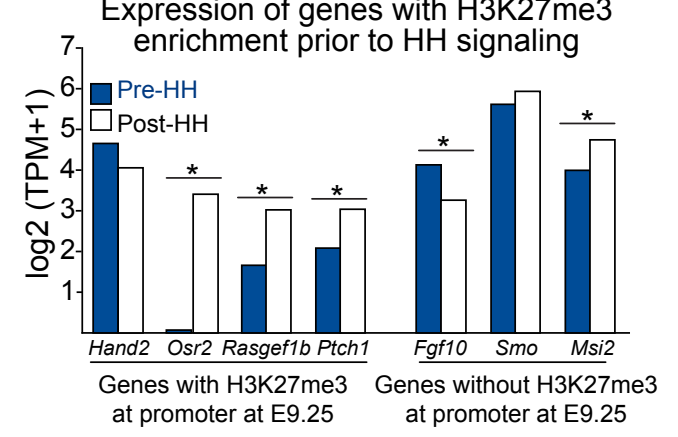

H3K27me3-

high gene expression

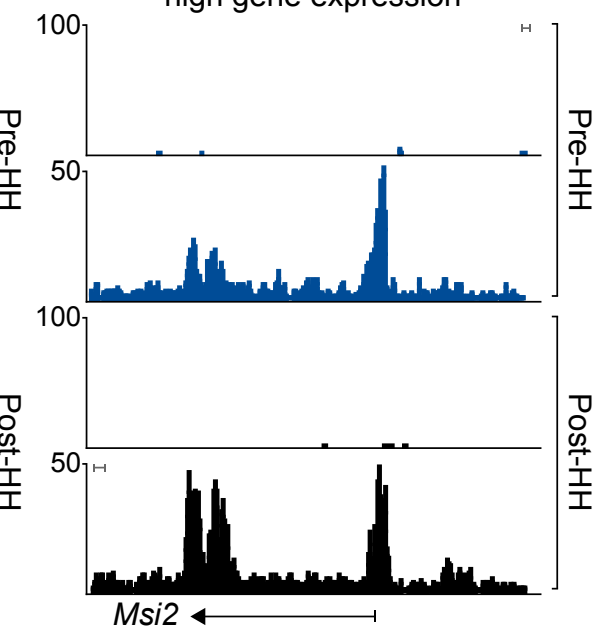

Figure S4. H3K27me3 is enriched at many $\mathrm{HH}$ target genes prior to $\mathrm{HH}$ signaling. $A, B$. $\mathrm{H} 3 \mathrm{~K} 27$ me3 enrichment at the promoters of predicted direct $\mathrm{HH}$ target gene promoters $(A)$ and $\mathrm{HH}$-responsive GBRs (B) in E9.25 and E10.5 anterior limb buds. C. Examples of H3K27me3 enrichment at promoters of $\mathrm{HH}$ target genes in E9.25 and anterior and posterior E10.5 limb buds. Orange shading indicates the binding regions for $\mathrm{HH}$-responsive GBRs defined in Figure S1C. D. Percentage of $\mathrm{HH}$ target gene promoters with $\mathrm{H} 3 \mathrm{~K} 27 \mathrm{me} 3$ and H3K27ac at E9.25 and E10.5. E. Relative expression levels of genes at E9.25 and E10.5 derived from RNA-seq data (Supplemental File 3) with and without H3K27me3 enrichment at promoters at E9.25. F-H. Examples of H3K27me3 and H3K27ac enrichment of genes with high expression at E9.25 with H3K27me3 enrichment (F), low expression with H3K27me3 enrichment (G) and high expression without H3K27me3 enrichment $(\mathrm{H})$. Scale bars for tracks indicate $1 \mathrm{~kb}$. 
A.

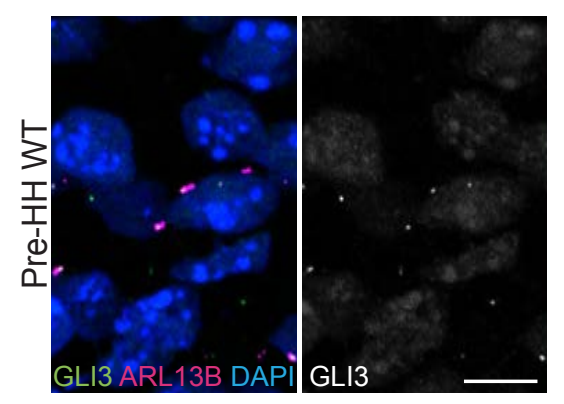

B.

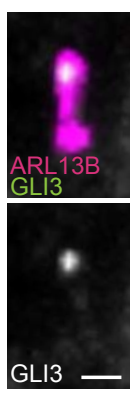

C.

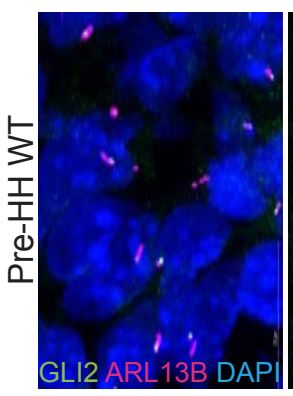

D.

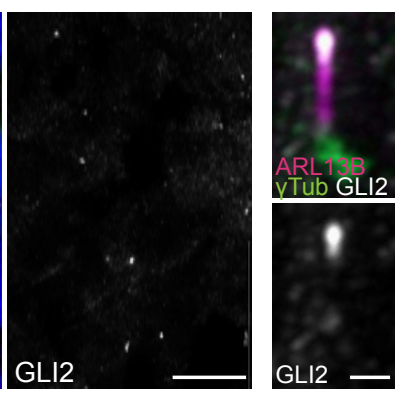

E.

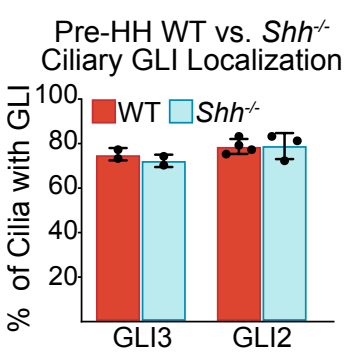

F.

Ciliary GLI3 Localization

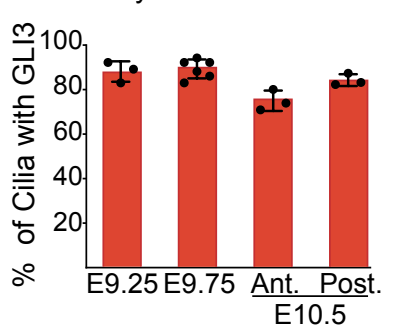

G.

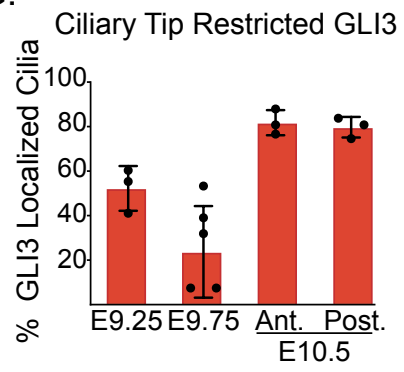

Figure S5. GLI ciliary distribution and localization in developing limbs. Images were collected with a Nikon A1R Resonant Scanning Confocal System and are maximum intensity projections. A,B. Endogenous GLI3 ${ }^{F L A G}$ and $A R L 13 b$ in a representative pre-HH E9.25 WT limb bud, and a representative cilium with GLI3 ${ }^{\mathrm{F} L A G}$ localization. C,D. Endogenous GLI2, ARL3b and X-TUBULIN (basal body) in a representative pre-HH E9.25 WT limb bud. GLI2 is localized to the opposite ciliary end as y-TUBULIN indicating most GLI localization is at the ciliary tips and not the base (D). E. Quantification of GLI2 and GLI3 ${ }^{F L A G}$ ciliary localization in E9.25 WT and Shh $h^{-1} \operatorname{limb}$ buds ( $n=2$ and 4 biological replicates, respectively). Note the similar levels of GLI2 and GLI3 ciliary localization in both genotypes, suggesting unprocessed GLI proteins are localized at the ciliary tip. F-G. Quantifications of GLI3 ciliary localization (D) in pre-HH E9.25 (21-23S; n=3), E9.75 (26-28S n=6) distal limb buds and E10.5 (35S; $\mathrm{n}=3$ ) anterior and posterior limb buds. E. Quantification of cilia in the same dataset with GLI3 restricted to the ciliary tip. Error bars in E-G indicate SEM. Scale bars for panels $A$ and $C$ indicate $10 \mu \mathrm{m}$; scale bards for panels $B$ and $D$ indicate $0.5 \mu \mathrm{m}$. 
A.

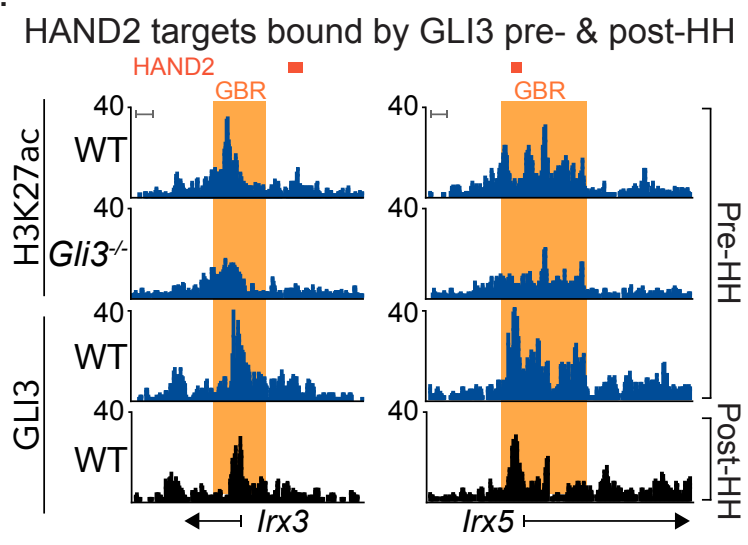

B.

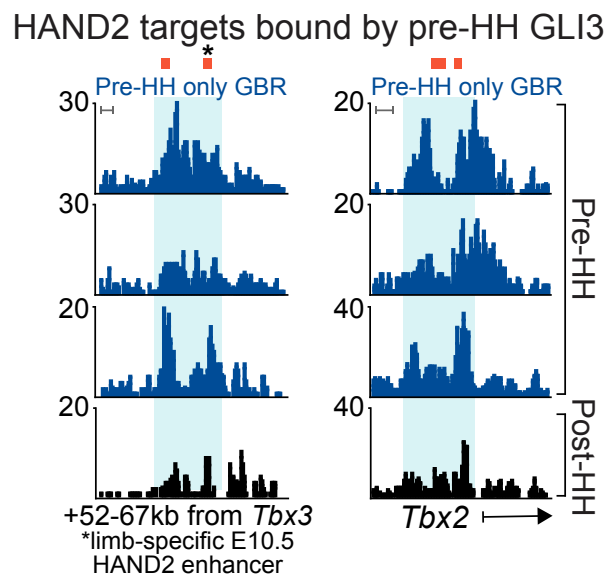

C.

Overlap of putative early limb-specific GLI3-HAND2 enhancers

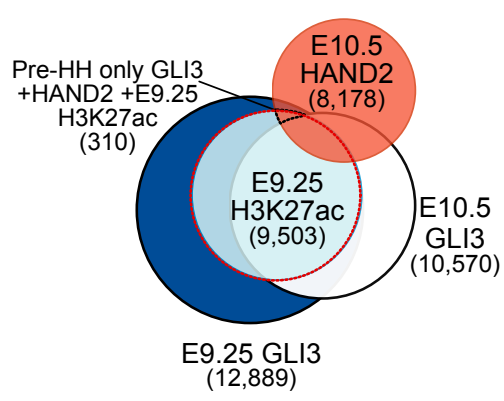

D.

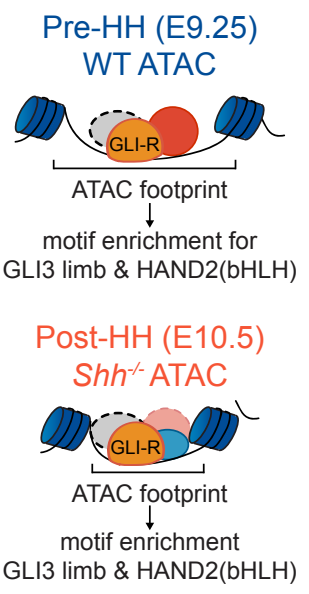

E.
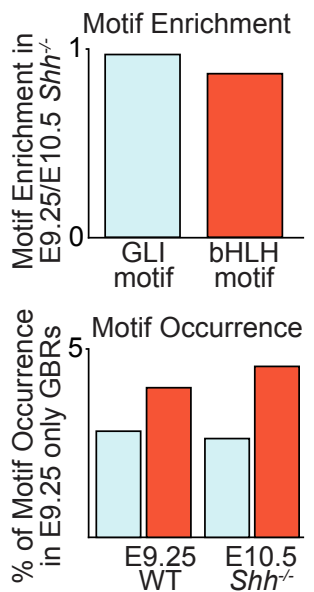

Figure S6. Co-localization of HAND2 and GLI3 in pre-HH limb buds. A. Examples of genes with HAND2 binding regions in E10.5 limbs (red bars denote Hand2 regions identified in Osterwalder et al, 2014) that are also bound by GLI3 (orange shading) in pre-HH or post-HH E10.5 limb buds. B. HAND2 target genes Tbx2 and Tbx3 have HAND2 bound regions that overlap with pre-HH specific GBRs (blue shading) but not post-HH GLI3 binding regions. Asterisks denote previously identified HAND2-bound enhancers (Osterwalder et al., 2014). C. Venn diagram of pre- and post-HH (E10.5) GLI3 binding regions, pre-HH H3K27ac peaks and previously identified E10.5 HAND2 limb binding sites (Osterwalder et al., 2014). 310 regions are bound by GLI3 only in pre-HH limb buds (not post-HH), overlap with HAND2 binding regions and are acetylated, indicating possibility of these regions being active enhancers. D. Schematic for performing motif enrichment in ATAC footprints identified in pre-HH WT limbs and compared to ATAC footprints in E10.5 Shh ${ }^{-/}$limbs. E. Quantification of enrichment (top) and occurrence (bottom) of limb GLI3 and face HAND2 motifs in the 310 acetylated pre-HH only GBRs overlapping HAND2 binding sites, compared to all acetylated pre-HH GBRs (red circle in D), for both pre-HH WT and post-HH E10.5 Shh ${ }^{-/}$limbs (Lex et al., 2020; Osterwalder et al., 2014). Note that GLI3 and HAND2 motif enrichment and occurrence are unchanged pre-HH WT and post-HH E10.5 Shh ${ }^{-/}$limbs. Scale bars denote $1 \mathrm{~kb}$. 